\title{
GLOBAL SOLUTIONS FOR THE TWO-COMPONENT CAMASSA-HOLM SYSTEM
}

\author{
KATRIN GRUNERT, HELGE HOLDEN, AND XAVIER RAYNAUD
}

\begin{abstract}
We prove existence of a global conservative solution of the Cauchy problem for the two-component Camassa-Holm $(2 \mathrm{CH})$ system on the line, allowing for nonvanishing and distinct asymptotics at plus and minus infinity. The solution is proven to be smooth as long as the density is bounded away from zero. Furthermore, we show that by taking the limit of vanishing density in the $2 \mathrm{CH}$ system, we obtain the global conservative solution of the (scalar) Camassa-Holm equation, which provides a novel way to define and obtain these solutions. Finally, it is shown that while solutions of the $2 \mathrm{CH}$ system have infinite speed of propagation, singularities travel with finite speed.
\end{abstract}

\section{INTRODUCTION}

The two-component Camassa-Holm $(2 \mathrm{CH})$ system, which was first derived in [23. Eq. (43)], is given by

$$
\begin{aligned}
u_{t}-u_{t x x}+\kappa u_{x}+3 u u_{x}-2 u_{x} u_{x x}-u u_{x x x}+\eta \rho \rho_{x} & =0, \\
\rho_{t}+(u \rho)_{x} & =0,
\end{aligned}
$$

for constants $\kappa \in \mathbb{R}$ and $\eta \in(0, \infty)$, or equivalently

$$
\begin{aligned}
u_{t}+u u_{x}+P_{x} & =0, \\
\rho_{t}+(u \rho)_{x} & =0,
\end{aligned}
$$

where $P$ is implicitly defined by

$$
P-P_{x x}=u^{2}+\kappa u+\frac{1}{2} u_{x}^{2}+\eta \frac{1}{2} \rho^{2} .
$$

We here study the Cauchy problem on the line where the equations (1.2)-(1.3) are augmented with initial conditions $\left.(u, \rho)\right|_{t=0}=\left(u_{0}, \rho_{0}\right)$. The equations have been derived as a model for shallow water by Constantin and Ivanov [5, where it is shown that $\eta$ positive and $\rho$ nonnegative is the physically relevant case.

The purpose of this paper is twofold: First of all we want to show the existence of a global and conservative solution of the $2 \mathrm{CH}$ system by suitable modifying recent results [9] for the (scalar) Camassa-Holm $(\mathrm{CH})$ equation

$$
u_{t}-u_{t x x}+\kappa u_{x}+3 u u_{x}-2 u_{x} u_{x x}-u u_{x x x}=0,
$$

Date: September 28, 2018.

2010 Mathematics Subject Classification. Primary: 35Q53, 35B35; Secondary: 35Q20.

Key words and phrases. Two-component Camassa-Holm equation, conservative solutions, Lipschitz continuous.

Research supported in part by the Research Council of Norway under projects Wavemaker, NoPiMa, and by the Austrian Science Fund (FWF) under Grant No. J3147. 
(which one obtains by taking $\rho$ identically zero in (1.2)-(1.3), or (1.1)). It turns out that the solution of (1.2)-1.3) is regular as long as the initial density $\rho_{0}$ is bounded away from zero (Theorem 6.1 and Corollary 6.2). This contrasts the case of the $\mathrm{CH}$ equation where one in general encounters weak solutions only. Secondly, we study the limit of the global conservative solution of the $2 \mathrm{CH}$ system for a sequence $\rho_{0}^{n}$ of initial densities tending to zero as $n \rightarrow \infty$. We find that the solution of the $2 \mathrm{CH}$ system approaches the global conservative solution of the CamassaHolm equation (Theorem 6.3). This offers an alternative approach to the study of conservative solutions, and a novel way to define conservative solutions. This is interesting because the $\mathrm{CH}$ equation enjoys two distinct classes of solutions, denoted dissipative and conservative solutions, respectively. In brief terms, the conservative solution preserves energy, while energy decreases for dissipative solutions. Both classes will in general have weak solutions rather than smooth solutions. To identify and characterize the two classes has turned out to be rather involved, see, e.g., 11, 2, 18, 19, 8, 10, and references therein. The approach in this paper characterizes conservative solutions as limits of smooth (classical) solutions of the $2 \mathrm{CH}$ system. This is novel.

The 2CH system (1.2)-1.3) has been studied extensively, from many different points of view, making a complete list of references too long. However, we here mention that Wang, Huang, and Chen 25] have studied conservative and global solutions of the $2 \mathrm{CH}$ system using a change of variables similar to the one employed here. The results here are more detailed and precise. In particular, we establish the semigroup property of the solutions and the continuity of the semigroup with respect to a new distance which is introduced. The vanishing density limit is not discussed in 25]. Escher, Lechtenfeld, and Yin [6] established a short-time existence theory for solutions using Kato techniques in the case $\eta=-1$ and $\kappa=0$. In the same paper it is shown that solutions may blow up in final time. Our approach does not apply to the case with $\eta$ negative. Constantin and Ivanov $[5$ showed that the solution for small initial data (or, more precisely, for $\rho_{0}$ close to a constant and small $u_{0}$ ) remains smooth. We here extend this result to data of arbitrary size, provided the density is bounded away from zero initially. A remarkable property of the system, which is shown in [13, is that when $\rho_{0}(x)>0$ for all $x \in \mathbb{R}$, the solution exists globally in time. In Theorem 6.1 we establish a local smoothing effect of the variable $\rho$, thereby extending the result of [13. In particular, we show how the characteristics govern the domain of smoothness.

For other related results pertaining to the present system, please see [13, 14, 15]. In addition to the $2 \mathrm{CH}$ system discussed in the present paper, there exists several other two-component generalizations of the $\mathrm{CH}$ equation, see, e.g., 3, 7, 11, 12, 16, 20, 24]. For traveling wave solutions see [5, 4, 21, 22,

Let us next describe the content of this paper more precisely. While we in this paper treat the case of arbitrary (nonvanishing) asymptotics of the initial data (and thereby of the solution), in the sense that

$$
\lim _{x \rightarrow \pm \infty} u_{0}(x)=u_{ \pm \infty} \quad \text { and } \quad \lim _{|x| \rightarrow \infty} \rho_{0}(x)=\rho_{\infty},
$$

we here, in order to make the presentation in the introduction more transparent, assume vanishing asymptotics for $u$, that is, $u_{ \pm \infty}=0$. Furthermore, we assume $\eta=1$ and $\kappa=0$. We first make a change from Eulerian to Lagrangian variables and introduce a new energy variable. The change of variables, which we now will detail, 
is related to the one used in [18] and, in particular, 9]. Assume that $u=u(x, t)$ is a solution, and define the characteristics $y=y(t, \xi)$ by

$$
y_{t}(t, \xi)=u(t, y(t, \xi))
$$

and the Lagrangian velocity by

$$
U(t, \xi)=u(t, y(t, \xi)) .
$$

By introducing the Lagrangian energy density $h$ and density $r$ by

$$
h(t, \xi)=u_{x}^{2}(t, y(t, \xi)) y_{\xi}(t, \xi)+\rho^{2}(t, y(t, \xi)) y_{\xi}(t, \xi), \quad r(t, \xi)=\rho(t, y(t, \xi)) y_{\xi}(t, \xi),
$$

we find that the system can be rewritten as

$$
\begin{aligned}
y_{t} & =U, \\
U_{t} & =-Q, \\
h_{t} & =2\left(U^{2}-P\right) U_{\xi}, \\
r_{t} & =0,
\end{aligned}
$$

where the functions $P$ and $Q$ are explicitly given by (3.12) and (3.13), respectively. We then establish the existence of a unique global solution for this system (see Theorem [3.6) which forms a continuous semigroup in an appropriate norm. In order to solve the Cauchy problem we have to choose the initial data appropriately. To accommodate for the possible concentration of energy we augment the natural initial data $u_{0}$ and $\rho_{0}$ with a nonnegative Radon measure $\mu_{0}$ such that the absolutely continuous part $\mu_{0, \text { ac }}$ is $\mu_{0, \text { ac }}=\left(u_{0, x}^{2}+\left(\rho_{0}-\rho_{0, \infty}\right)^{2}\right) d x$. The precise translation of these initial data is given in Theorem 4.9. One then solves the system in Lagrangian coordinates. The translation back to Eulerian variables is described in Theorem 4.10. However, there is an intrinsic problem in this latter translation if one wants a continuous semigroup. This is due to the problem of relabeling; to each solution in Eulerian variables there exist several distinct solutions in Lagrangian variables as there are additional degrees of freedom in the Lagrangian variables. In order to resolve this issue to get a continuous semigroup, one has to identify Lagrangian functions corresponding to one and the same Eulerian solution. This is treated in Sec. 4. The main existence theorem, Theorem 5.2, states that for $u_{0} \in H^{1}$ and $\rho_{0} \in L^{2}$ and $\mu_{0}$ a nonnegative Radon measure with absolutely continuous part $\mu_{0, \text { ac }}$ such that $\mu_{0, \text { ac }}=\left(u_{0, x}^{2}+\left(\rho_{0}-\rho_{0, \infty}\right)^{2}\right) d x$, there exists a continuous semigroup $T_{t}$ such that $(u, \rho, \mu)(t)=T_{t}\left(u_{0}, \rho_{0}, \mu_{0}\right)$ is a weak global and conservative solution of the $2 \mathrm{CH}$ system. In addition, the measure $\mu$ satisfies

$$
\left(u^{2}+\mu\right)_{t}+\left(u\left(u^{2}+\mu\right)\right)_{x}=\left(u^{3}-2 P u\right)_{x},
$$

weakly. Furthermore, for almost all times the measure $\mu$ is absolutely continuous and $\mu=\left(u_{x}^{2}+\left(\rho-\rho_{\infty}\right)^{2}\right) d x$.

In order to analyze the case where we consider a sequence of initial densities $\rho_{0}^{n}$ tending to zero as $n \rightarrow \infty$, we need to have a sufficiently strong stability result. To that effect, we have the following result, Theorem 6.3. Consider a sequence of initial data $\left(u_{0}^{n}, \rho_{0}^{n}, \mu_{0}^{n}\right)$ such that $u_{0}^{n} \rightarrow u_{0}$ in $H^{1}(\mathbb{R}), \rho_{0}^{n}-\rho_{0, \infty}^{n} \rightarrow 0$ in $L^{2}(\mathbb{R}), \rho_{0, \infty}^{n} \rightarrow 0$, with $\rho_{0}^{n} \geq d_{n}>0$ for all $n$. Assume that the initial measure is absolutely continuous, that is, $\mu_{0}^{n}=\mu_{0, \text { ac }}^{n}=\left(\left(u_{0, x}^{n}\right)^{2}+\left(\rho_{0}^{n}-\rho_{0, \infty}^{n}\right)^{2}\right) d x$. Then the sequence $u^{n}(t)$ will converge in $L^{\infty}(\mathbb{R})$ to the weak, conservative global solution of the Camassa-Holm equation with initial data $u_{0}$. 
Finally, we want to address the regularity issue. Consider an open subset I of $\mathbb{R}$. We say that $(u, \rho, \mu)$ is $p$-regular on $I$ if

$$
u \in W^{p, \infty}(I), \rho \in W^{p-1, \infty}(I) \text { and } \mu_{\mathrm{ac}}=\mu \text { on } I .
$$

A surprising feature of the $2 \mathrm{CH}$ system is that while it has an infinite speed of propagation [17, singularities travel with finite speed. This is the content of the following theorem, Theorem 6.1] Assume that the initial data $\left(u_{0}, \rho_{0}, \mu_{0}\right)$ is $p$ regular on an interval $\left(x_{0}, x_{1}\right)$ such that $\rho_{0}^{2} \geq c>0$ on $\left(x_{0}, x_{1}\right)$. Then the solution $(u, \rho, \mu)(t)$ is $p$-regular on the interval given by the characteristics emanating from $\left(x_{0}, x_{1}\right)$. More precisely, it is $p$-regular on the interval $\left(y\left(t, \xi_{0}\right), y\left(t, \xi_{1}\right)\right)$, where $\xi_{0}$ and $\xi_{1}$ satisfy $y\left(0, \xi_{0}\right)=x_{0}$ and $y\left(0, \xi_{1}\right)=x_{1}$ and are defined as

$$
\xi_{0}=\sup \left\{\xi \in \mathbb{R} \mid y(0, \xi) \leq x_{0}\right\} \text { and } \xi_{1}=\inf \left\{\xi \in \mathbb{R} \mid y(0, \xi) \geq x_{1}\right\} .
$$

Thus we see that regularity is preserved between characteristics.

\section{EUlerian COORDinAtes}

We consider the Cauchy problem for the two-component Camassa-Holm system with arbitrary $\kappa \in \mathbb{R}$ and $\eta \in(0, \infty)$,

$$
\begin{aligned}
u_{t}-u_{t x x}+\kappa u_{x}+3 u u_{x}-2 u_{x} u_{x x}-u u_{x x x}+\eta \rho \rho_{x} & =0, \\
\rho_{t}+(u \rho)_{x} & =0,
\end{aligned}
$$

with initial data $\left.u\right|_{t=0}=u_{0}$ and $\left.\rho\right|_{t=0}=\rho_{0}$. We are interested in global solutions for initial data $u_{0}$ with nonvanishing and possibly distinct limits at infinity, that is,

$$
\lim _{x \rightarrow-\infty} u_{0}(x)=u_{-\infty} \quad \text { and } \quad \lim _{x \rightarrow \infty} u_{0}(x)=u_{\infty}
$$

Furthermore we assume that the initial density has equal asymptotics which need not to be zero, that is,

$$
\lim _{x \rightarrow \pm \infty} \rho_{0}(x)=\rho_{\infty}
$$

More precisely we introduce the spaces

$H_{\infty}(\mathbb{R})=\left\{v \in L_{\text {loc }}^{1}(\mathbb{R}) \mid v(x)=\bar{v}(x)+v_{-\infty} \chi(-x)+v_{\infty} \chi(x), \bar{v} \in H^{1}(\mathbb{R}), v_{ \pm \infty} \in \mathbb{R}\right\}$,

where $\chi$ denotes a smooth partition function with support in $[0, \infty)$ such that $\chi(x)=1$ for $x \geq 1$ and $\chi^{\prime}(x) \geq 0$ for $x \in \mathbb{R}$, and

$$
L_{\text {const }}^{2}(\mathbb{R})=\left\{g \in L_{\text {loc }}^{1}(\mathbb{R}) \mid g(x)=g_{\infty}+\bar{g}(x), \bar{g} \in L^{2}(\mathbb{R}), g_{\infty} \in \mathbb{R}\right\} .
$$

Subsequently, we will assume that

$$
u_{0} \in H_{\infty}(\mathbb{R}), \quad \rho_{0} \in L_{\text {const }}^{2}(\mathbb{R}) .
$$

Introducing the mapping $I_{\chi}$ from $H^{1}(\mathbb{R}) \times \mathbb{R}^{2}$ into $H_{\text {loc }}^{1}(\mathbb{R})$ given by

$$
I_{\chi}\left(\bar{u}, c_{-}, c_{+}\right)(x)=\bar{u}(x)+c_{-} \chi(-x)+c_{+} \chi(x)
$$

for any $\left(\bar{u}, c_{-}, c_{+}\right) \in H^{1}(\mathbb{R}) \times \mathbb{R}^{2}$, yields that any initial condition $u_{0} \in H_{\infty}(\mathbb{R})$ is defined by an element in $H^{1}(\mathbb{R}) \times \mathbb{R}^{2}$ through the mapping $I_{\chi}$. Hence we see that $H_{\infty}(\mathbb{R})$ is the image of $H^{1}(\mathbb{R}) \times \mathbb{R}^{2}$ by $I_{\chi}$, that is, $H_{\infty}(\mathbb{R})=I_{\chi}\left(H^{1}(\mathbb{R}) \times \mathbb{R}^{2}\right)$. The linear mapping $I_{\chi}$ is injective. We equip $H_{\infty}(\mathbb{R})$ with the norm

$$
\|u\|_{H_{\infty}}=\|\bar{u}\|_{H^{1}}+\left|c_{-}\right|+\left|c_{+}\right|
$$


where $u=I_{\chi}\left(\bar{u}, c_{-}, c_{+}\right)$. Then $H_{\infty}(\mathbb{R})$ is a Banach space. Given another partition function $\tilde{\chi}$, we define the mapping $\left(\tilde{\bar{u}}, \tilde{c}_{-}, \tilde{c}_{+}\right)=\Psi\left(\bar{u}, c_{-}, c_{+}\right)$from $H^{1}(\mathbb{R}) \times \mathbb{R}^{2}$ to $H^{1}(\mathbb{R}) \times \mathbb{R}^{2}$ as $\tilde{c}_{-}=c_{-}, \tilde{c}_{+}=c_{+}$and

$$
\tilde{\bar{u}}(x)=\bar{u}(x)+c_{-}(\chi(-x)-\tilde{\chi}(-x))+c_{+}(\chi(x)-\tilde{\chi}(x)) .
$$

The linear mapping $\Psi$ is a continuous bijection. Since

$$
I_{\chi}=I_{\tilde{\chi}} \circ \Psi,
$$

we can see that the definition of the Banach space $H_{\infty}(\mathbb{R})$ does not depend on the choice of the partition function $\chi$. The norm defined by (2.7) for different partition functions $\chi$ are all equivalent.

Similarly, one can associate to any element $\rho \in L_{\text {const }}^{2}(\mathbb{R})$ the unique pair $(\bar{\rho}, k) \in$ $L^{2}(\mathbb{R}) \times \mathbb{R}$ through the mapping $J$ from $L^{2}(\mathbb{R}) \times \mathbb{R}$ to $L_{\text {const }}^{2}(\mathbb{R})$ which is defined as

$$
J(\bar{\rho}, k)=\bar{\rho}+k .
$$

In fact $J$ is bijective from $L^{2}(\mathbb{R}) \times \mathbb{R}$ to $L_{\text {const }}^{2}(\mathbb{R})$, which allows us to equip $L_{\text {const }}^{2}(\mathbb{R})$ with the norm

$$
\|\rho\|_{L_{\text {const }}^{2}}=\|\bar{\rho}\|_{L^{2}}+|k|,
$$

where we decomposed $\rho$ according to $\rho=J(\bar{\rho}, k)$. Thus $L_{\text {const }}^{2}(\mathbb{R})$ together with the norm defined in (2.10) is a Banach space.

Note that for smooth solutions, we have the following conservation law

$$
\left(u^{2}+u_{x}^{2}+\eta \rho^{2}\right)_{t}+\left(u\left(u^{2}+u_{x}^{2}+\eta \rho^{2}\right)\right)_{x}=\left(u^{3}+\kappa u^{2}-2 P u\right)_{x} .
$$

Moreover, if $(u(t, x), \rho(t, x))$ is a solutions of the two-component Camassa-Holm system (2.1), then, for any constant $\alpha \in \mathbb{R}$ we easily find that

$$
v(t, x)=u(t, x-\alpha t)+\alpha, \quad \text { and } \quad \tau(t, x)=\sqrt{\eta} \rho(t, x-\alpha t),
$$

solves the two-component Camassa-Holm system with $\kappa$ replaced by $\kappa-2 \alpha$ and $\eta=1$. Therefore, without loss of generality, we assume in what follows, that $\lim _{x \rightarrow-\infty} u_{0}(x)=0$ and $\eta=1$. In addition, we only consider the case $\kappa=0$ as one can make the same conclusions for $\kappa \neq 0$ with slight modifications.

\section{Global solutions in Lagrangian CoOrdinates}

The aim of this section is to rewrite the two-component Camassa-Holm system as a system of ordinary differential equations in a suitable Banach space, such that we can prove global existence of solutions therefore.

3.1. Equivalent system. Rewrite the two-component Camassa-Holm system as the following system

$$
\begin{aligned}
u_{t}+u u_{x}+P_{x} & =0, \\
\rho_{t}+(u \rho)_{x} & =0,
\end{aligned}
$$

where $P$ is implicitly defined by

$$
P-P_{x x}=u^{2}+\frac{1}{2} u_{x}^{2}+\frac{1}{2} \rho^{2} .
$$

Introduce the subspace $H_{0, \infty}(\mathbb{R})$ of $H_{\infty}(\mathbb{R})$ as

$$
H_{0, \infty}(\mathbb{R})=I_{\chi}\left(H^{1}(\mathbb{R}) \times\{0\} \times \mathbb{R}\right) .
$$

\footnotetext{
${ }^{1}$ For $\kappa$ nonzero 3.2 is simply replaced by $P-P_{x x}=u^{2}+\kappa u+\frac{1}{2} u_{x}^{2}+\frac{1}{2} \rho^{2}$.
} 
Then we obtain, using (3.2), under the assumption that $\rho \in L_{\text {const }}^{2}(\mathbb{R})$ and $u \in$ $H_{0, \infty}(\mathbb{R})$ that the function $P$ can be expressed through

$$
\begin{aligned}
P(x)= & c^{2} \chi^{2}(x)+\frac{1}{2} \int_{\mathbb{R}} e^{-|x-z|}\left(2 c \chi \bar{u}+\bar{u}^{2}+\frac{1}{2} u_{x}^{2}+2 c^{2} \chi^{\prime 2}+2 c^{2} \chi \chi^{\prime \prime}\right)(z) d z \\
& +\frac{1}{2} k^{2}+\frac{1}{2} \int_{\mathbb{R}} e^{-|x-z|}\left(\frac{1}{2} \bar{\rho}^{2}+k \bar{\rho}\right)(z) d z .
\end{aligned}
$$

In particular, $P \in H_{\infty}(\mathbb{R})$ and especially $P_{x} \in L^{2}(\mathbb{R})$.

We introduce the Lagrangian variables and rewrite the governing equations (3.1) with respect to these variables. Let the characteristics $y(t, \xi)$, which can be decomposed as $y(t, \xi)=\zeta(t, \xi)+\xi$, be defined as the solutions of

$$
y_{t}(t, \xi)=u(t, y(t, \xi))
$$

for some given initial function $y(0, \xi)$. We define

$$
U(t, \xi)=u(t, y(t, \xi))
$$

which can be decomposed as

$$
U(t, \xi)=\bar{U}(t, \xi)+c(t) \chi \circ y(t, \xi),
$$

where $\bar{U} \in H^{1}(\mathbb{R})$ and $c \in \mathbb{R}$. In addition, we define $h \in L^{2}(\mathbb{R})$ formally as

$$
h(t, \xi)=u_{x}^{2}(t, y(t, \xi)) y_{\xi}(t, \xi)+\bar{\rho}^{2}(t, y(t, \xi)) y_{\xi}(t, \xi)
$$

so that $\left(u_{x}^{2}(t, x)+\bar{\rho}^{2}(t, x)\right) d x=y_{\#}(h(t, \xi) d \xi)$, and $r \in L_{\text {const }}^{2}(\mathbb{R})$ as

$$
r(t, \xi)=\rho(t, y(t, \xi)) y_{\xi}(t, \xi)
$$

Moreover, $r$ can be decomposed as

$$
r(t, \xi)=\bar{r}(t, \xi)+k(t) y_{\xi}(t, \xi),
$$

so that $\rho(t, x) d x=k(t)+y_{\#}(\bar{r}(t, \xi) d \xi)$. Here $y_{\#}$ denotes the pushforward We have

$$
y_{\xi} h=U_{\xi}^{2}+\bar{r}^{2} .
$$

The system (3.1)-3.2) rewrites

$$
\begin{aligned}
y_{t} & =U, \\
U_{t} & =-Q, \\
c_{t} & =0, \\
h_{t} & =2\left(U^{2}+\frac{1}{2} k^{2}-P\right) U_{\xi}, \\
r_{t} & =0, \\
k_{t} & =0,
\end{aligned}
$$

where the functions $P(t, \xi)$ and $Q(t, \xi)$ are given by

$P(\xi)=\frac{1}{4} \int_{\mathbb{R}} e^{-|y(\xi)-y(\eta)|}\left(\left(2 \bar{U}^{2}+4 c \bar{U} \chi \circ y\right) y_{\xi}+2 k \bar{r}+h\right)(\eta) d \eta+c^{2} g \circ y(\xi)+\frac{1}{2} k^{2}$,

\footnotetext{
${ }^{2}$ The push-forward of a measure $\nu$ by a measurable function $f$ is the measure $f_{\#} \nu$ defined as $f_{\#} \nu(B)=\nu\left(f^{-1}(B)\right)$ for any Borel set $B$.
} 
and

$Q(\xi)=-\frac{1}{4} \int_{\mathbb{R}} \operatorname{sign}(\xi-\eta) e^{-|y(\xi)-y(\eta)|}\left(\left(2 \bar{U}^{2}+4 c \bar{U} \chi \circ y\right) y_{\xi}+2 k \bar{r}+h\right)(\eta) d \eta+c^{2} g^{\prime} \circ y(\xi)$,

where

$$
g(x)=\chi^{2}(x)+\frac{1}{2} \int_{\mathbb{R}} e^{-|x-z|}\left(2 \chi^{\prime 2}+2 \chi \chi^{\prime \prime}\right)(z) d z .
$$

Equations (3.11a), (3.11b), (3.11d), and (3.11e), follow directly from (3.1)-(3.2). The value of the energy density $\left(u^{2}+u_{x}^{2}+\rho^{2}\right.$ exit) is essentially controlled by the variable $h$, and the evolution equation for $h$, (3.11d , follows from the conservation law (2.11), which yields

$$
\left(u_{x}^{2}+\rho^{2}\right)_{t}+\left(u\left(u_{x}^{2}+\rho^{2}\right)\right)_{x}=2\left(u^{2}-P\right) u_{x} .
$$

Equations (3.11c) and (3.11f state that the asymptotics do not evolve in time. Formally, it comes from the fact that, if all functions are smooth and the derivatives decay sufficiently fast, then

$$
\lim _{x \rightarrow \pm \infty} u_{t}(x)=-\lim _{x \rightarrow \pm \infty}\left(u u_{x}+P_{x}\right)=0
$$

and

$$
\lim _{x \rightarrow \pm \infty} \rho_{t}(x)=-\lim _{x \rightarrow \pm \infty}\left(\rho u_{x}+u \rho_{x}\right)=0 .
$$

The variables $y, U$, and $r$ given by (3.4), (3.5) and been as the standard Lagrangian variables for the equation. However, to carry out our analysis we need to introduce the variables $\zeta, \bar{U}$ and $\bar{r}$, which are derived from the former variables and, in addition, belong to the appropriate Banach spaces. In those variables the system of governing equation writes

$$
\begin{aligned}
\zeta_{t} & =U, \\
\bar{U}_{t} & =-Q-c\left(\chi^{\prime} \circ y\right) U, \\
h_{t} & =2\left(U^{2}+\frac{1}{2} k^{2}-P\right) U_{\xi}, \\
\bar{r}_{t} & =-k U_{\xi}, \\
c_{t} & =0, \\
k_{t} & =0 .
\end{aligned}
$$

3.2. Existence and uniqueness of solutions of the equivalent system. Let $V$ be the Banach space defined by

$$
V=\left\{f \in C_{b}(\mathbb{R}) \mid f_{\xi} \in L^{2}(\mathbb{R})\right\}
$$

where $C_{b}(\mathbb{R})=C(\mathbb{R}) \cap L^{\infty}(\mathbb{R})$ and the norm of $V$ is given by $\|f\|_{V}=\|f\|_{L^{\infty}}+$ $\left\|f_{\xi}\right\|_{L^{2}}$. We define the Banach space $E$ by

$$
E=V \times H_{0, \infty}(\mathbb{R}) \times L^{2}(\mathbb{R}) \times L_{\text {const }}^{2}(\mathbb{R}),
$$

and

$$
\bar{E}=V \times H^{1}(\mathbb{R}) \times \mathbb{R} \times L^{2}(\mathbb{R}) \times L^{2}(\mathbb{R}) \times \mathbb{R} .
$$

Then $E$ is in isometry with $\bar{E}$ and we define

$$
\|(\zeta, U, h, r)\|_{E}=\left\|\left(\zeta, I_{\chi}^{-1}(U), h, J^{-1}(r)\right)\right\|_{\bar{E}} .
$$


However, all partition functions give rise to equivalent norms (cf. [9, Sec. 6]). For convenience, we will often abuse the notations and denote by the same $X$ the two elements $(y, \bar{U}, c, h, \bar{r}, k)$ and $(y, U, h, r)$ where, by definition, $U=\bar{U}+c \chi \circ y$ and $r=\bar{r}+k y_{\xi}$. Furthermore, we will occasionally write $(\zeta, \bar{U}, c, h, \bar{r}, k)$ and $(\zeta, U, h, r)$ for $X$.

The investigation in [9, Sec. 3] of $g$ defined by (3.14) showed that $g \in H_{0, \infty}(\mathbb{R})$ with $\lim _{x \rightarrow \infty} g(x)=1$. Moreover, $g$ is monotonically increasing and $g^{\prime} \in H^{1}(\mathbb{R})$.

This yields the following result.

Lemma 3.1. For any $X=(\zeta, U, h, r)$ in $E$, we define the maps $\mathcal{Q}$ and $\mathcal{P}$ as $\mathcal{Q}(X)=Q$ and $\mathcal{P}(X)=P$, where $P$ and $Q$ are given by (3.12) and (3.13), respectively. Then, $\mathcal{P}-\frac{1}{2} k^{2}-U^{2}$ is a Lipschitz map on bounded sets from $E$ to $H^{1}(\mathbb{R})$ and $\mathcal{Q}$ is a Lipschitz map on bounded sets from $E$ to $H^{1}(\mathbb{R})$. Moreover we have

$$
\begin{gathered}
Q_{\xi}=-\frac{1}{2} h-\left(U^{2}+\frac{1}{2} k^{2}-P\right) y_{\xi}-k \bar{r}, \\
P_{\xi}=Q\left(1+\zeta_{\xi}\right) .
\end{gathered}
$$

Proof. The structure of the involved functions is similar to the ones in 9 . We therefore refer to the proof of [9, Lemma 3.1].

Theorem 3.2. Given $X_{0}=\left(\zeta_{0}, U_{0}, h_{0}, r_{0}\right) \in E$, then there exists a time $T$ depending only on $\left\|X_{0}\right\|_{E}$ such that (3.15) admits a unique solution in $C^{1}([0, T], E)$ with initial data $X_{0}$.

Proof. Solutions of (3.15) can be rewritten as

$$
X(t)=X_{0}+\int_{0}^{t} F(X(\tau)) d \tau,
$$

where $F: E \rightarrow E$ is defined by the right-hand side of (3.15). The integrals are defined as Riemann integrals of continuous functions on the Banach space $E$. Using Lemma 3.1 we can check that $F(X)$ is a Lipschitz function on bounded sets of $E$. Since $E$ is a Banach space, we use the standard contraction argument to prove the theorem.

Differentiating (3.11) and (3.15), we obtain

$$
\begin{aligned}
y_{\xi, t} & =U_{\xi}, \\
U_{\xi, t} & =\frac{1}{2} h+\left(U^{2}+\frac{1}{2} k^{2}-P\right) y_{\xi}+k \bar{r}, \\
h_{t} & =2\left(U^{2}+\frac{1}{2} k^{2}-P\right) U_{\xi}, \\
r_{t} & =0,
\end{aligned}
$$

and

$$
\begin{aligned}
\zeta_{\xi, t} & =U_{\xi}, \\
\bar{U}_{\xi, t} & =\frac{1}{2} h+\left(U^{2}+\frac{1}{2} k^{2}-P\right) y_{\xi}+k \bar{r}-c \chi^{\prime \prime} \circ y y_{\xi} U-c \chi^{\prime} \circ y U_{\xi}, \\
\bar{r}_{t} & =-k U_{\xi} .
\end{aligned}
$$

We now turn to the proof of the existence of global solutions of 3.15). To that end we consider initial data that belongs to $E \cap\left(\left[W^{1, \infty}(\mathbb{R})\right]^{2} \times\left[L^{\infty}(\mathbb{R})\right]^{2}\right)$, where 
$W^{1, \infty}(\mathbb{R})=\left\{f \in C_{b}(\mathbb{R}) \mid f_{\xi} \in L^{\infty}(\mathbb{R})\right\}$. Given $\left(\zeta_{0}, U_{0}, h_{0}, r_{0}\right) \in E \cap\left(\left[W^{1, \infty}(\mathbb{R})\right]^{2} \times\right.$ $\left.\left[L^{\infty}(\mathbb{R})\right]^{2}\right)$, we consider the short-time solutions $(\zeta, U, h, r) \in C([0, T], E)$ of (3.15) given by Theorem 3.2 . Using the fact that $\mathcal{Q}$ and $\mathcal{P}-\frac{1}{2} k^{2}-U^{2}$ are Lipschitz on bounded sets and, since $X \in C([0, T], E)$, we can prove that $P-\frac{1}{2} k^{2}-U^{2}$ and $Q$ belong to $C\left([0, T], H^{1}(\mathbb{R})\right)$. Hence we now consider $P-\frac{1}{2} k^{2}-U^{2}$ and $Q$ as given functions in $C\left([0, T], H^{1}(\mathbb{R})\right)$. Then, for any fixed $\xi \in \mathbb{R}$, we can solve the system of ordinary differential equations

$$
\begin{aligned}
\frac{d}{d t} \alpha(t, \xi) & =\beta(t, \xi) \\
\frac{d}{d t} \beta(t, \xi) & =\frac{1}{2} \gamma(t, \xi)+\left[\left(U^{2}+\frac{1}{2} k^{2}-P\right)(t, \xi)\right](1+\alpha(t, \xi))+k \delta(t, \xi), \\
\frac{d}{d t} \gamma(t, \xi) & =2\left(U^{2}+\frac{1}{2} k^{2}-P\right) \beta(t, \xi), \\
\frac{d}{d t} \delta(t, \xi) & =-k \beta(t, \xi)
\end{aligned}
$$

which is obtained by substituting $\zeta_{\xi}, U_{\xi}, h$, and $\bar{r}$ in (3.20) by the unknowns $\alpha$, $\beta, \gamma$, and $\delta$, respectively in $\left[L^{\infty}(\mathbb{R})\right]^{4}$. We have to specify the initial conditions for (3.21).

Let $\mathcal{A}$ be the following set

$\mathcal{A}=\left\{\xi \in \mathbb{R}|| \zeta_{0, \xi}\left|\leq\left\|\zeta_{0, \xi}\right\|_{L^{\infty}},\right| U_{0, \xi}(\xi)\left|\leq\left\|U_{0, \xi}\right\|_{L^{\infty}},\right| h_{0}\left|\leq\left\|h_{0}\right\|_{L^{\infty}},\right| r_{0} \mid \leq\left\|r_{0}\right\|_{L^{\infty}}\right\}$.

By assumption we have that $\mathcal{A}$ has full measure, that is, $\operatorname{meas}\left(\mathcal{A}^{c}\right)=0$. For $\xi \in \mathcal{A}$ we define $(\alpha(0, \xi), \beta(0, \xi), \gamma(0, \xi), \delta(0, \xi))=\left(\zeta_{0, \xi}(\xi), U_{0, \xi}(\xi), h_{0}(\xi), \bar{r}_{0}(\xi)\right)$. However, if $\xi \in \mathcal{A}^{c}$, we set $(\alpha(0, \xi), \beta(0, \xi), \gamma(0, \xi), \delta(0, \xi))=(0,0,0,0)$.

Lemma 3.3. Given some initial condition $X_{0}=\left(\zeta_{0}, U_{0}, h_{0}, r_{0}\right) \in E \cap\left(\left[W^{1, \infty}(\mathbb{R})\right]^{2} \times\right.$ $\left.\left[L^{\infty}(\mathbb{R})\right]^{2}\right)$, we consider the solution $X=(\zeta, U, h, r) \in C^{1}([0, T], E)$ of (3.15) given by Theorem 3.2. Then $X \in C^{1}\left([0, T], E \cap\left(\left[W^{1, \infty}(\mathbb{R})\right]^{2} \times\left[L^{\infty}(\mathbb{R})\right]^{2}\right)\right)$. The functions $\alpha(t, \xi), \beta(t, \xi), \gamma(t, \xi)$, and $\delta(t, \xi)$ which are obtained by solving (3.21) for any fixed given $\xi$ with the initial condition specified above, coincide for almost every $\xi$ and for all times $t$ with $\zeta_{\xi}, U_{\xi}$, h, and $\bar{r}$, respectively, that is, for all $t \in[0, T]$, we have

$$
(\alpha(t, \xi), \beta(t, \xi), \gamma(t, \xi), \delta(t, \xi))=\left(\zeta_{\xi}(t, \xi), U_{\xi}(t, \xi), h(t, \xi), \bar{r}(t, \xi)\right)
$$

for almost every $\xi \in \mathbb{R}$.

Thus this lemma allows us to pick a special representative for $\left(\zeta_{\xi}, U_{\xi}, h, \bar{r}\right)$ given by $(\alpha, \beta, \gamma, \delta)$, which is defined for all $\xi \in \mathbb{R}$ and which, for any given $\xi$, satisfies the ordinary differential equation (3.21). In what follows we will of course identify the two and set $\left(\zeta_{\xi}, U_{\xi}, h, \bar{r}\right)$ equal to $(\alpha, \beta, \gamma, \delta)$.

Proof. The proof is similar to the one of [18, Lemma 2.4] and hence we refer the interested reader there.

We define the set $\mathcal{G}$ as follows.

Definition 3.4. The set $\mathcal{G}$ is composed of all $(\zeta, U, h, r) \in E$ such that

$$
\begin{aligned}
& (\zeta, U, h, r) \in\left[W^{1, \infty}(\mathbb{R})\right]^{2} \times\left[L^{\infty}(\mathbb{R})\right]^{2}, \\
& y_{\xi} \geq 0, h \geq 0, y_{\xi}+h>0 \text { almost everywhere, } \\
& y_{\xi} h=U_{\xi}^{2}+\bar{r}^{2} \text { almost everywhere, }
\end{aligned}
$$

where we denote $y(\xi)=\zeta(\xi)+\xi$. 
The set $\mathcal{G}$ is preserved by the flow and for initial data in $\mathcal{G}$ we obtain global solutions.

Lemma 3.5. Given initial data $X_{0}=\left(\zeta_{0}, U_{0}, h_{0}, r_{0}\right)$ in $\mathcal{G}$, let $X(t)=(\zeta(t), U(t), h(t), r(t))$ be the short-time solution of (3.15) in $C^{1}([0, T], E)$ for some $T>0$ with initial data $\left(\zeta_{0}, U_{0}, h_{0}, r_{0}\right)$. Then

(i) $X(t)$ belongs to $\mathcal{G}$ for all $t \in[0, T]$,

(ii) for almost every $t \in[0, T]$, we have $y_{\xi}(t, \xi)>0$ for almost every $\xi \in \mathbb{R}$.

Proof. The proof follows the same lines as in [18, Lemma 2.7]. We prove only (3.23c). From the governing equations, for any given $\xi \in \mathbb{R}$, we get

and

$$
\begin{gathered}
\frac{d}{d t}\left(y_{\xi} h\right)=U_{\xi} h+2 y_{\xi}\left(U^{2}+\frac{1}{2} k^{2}-P\right) U_{\xi}, \\
\frac{d}{d t} U_{\xi}^{2}=2 U_{\xi}\left(\frac{1}{2} h+\left(U^{2}+\frac{1}{2} k^{2}-P\right) y_{\xi}+k \bar{r}\right)
\end{gathered}
$$

$$
\frac{d}{d t} \bar{r}^{2}=-2 k \bar{r} U_{\xi}
$$

Hence, $\frac{d}{d t}\left(y_{\xi} h-U_{\xi}^{2}-\bar{r}^{2}\right)=0$ and (3.23c) is proved.

We are now ready to prove global existence of solutions to (3.15).

Theorem 3.6. For any $X_{0}=\left(y_{0}, U_{0}, h_{0}, r_{0}\right) \in \mathcal{G}$, the system 3.15 admits a unique global solution $X(t)=(y(t), U(t), h(t), r(t))$ in $C^{1}\left(\mathbb{R}_{+}, E\right)$ with initial data $X_{0}=\left(y_{0}, U_{0}, h_{0}, r_{0}\right)$. We have $X(t) \in \mathcal{G}$ for all times. If we equip $\mathcal{G}$ with the topology inducted by the E-norm, then the mapping $S: \mathcal{G} \times \mathbb{R}_{+} \rightarrow \mathcal{G}$ defined as

$$
S_{t}(\bar{X})=X(t)
$$

is a continuous semigroup. More precisely, given $M>0$ and $T>0$, there exists a constant $C_{M}$ which only depends on $M$ and $T$ such that, for any two elements $X_{\alpha}$, $X_{\beta} \in \mathcal{G}$ such that $\left\|X_{\alpha}\right\|_{E} \leq M,\left\|X_{\beta}\right\|_{E} \leq M$, we have

$$
\left\|S_{t} X_{\alpha}-S_{t} X_{\beta}\right\| \leq C_{M}\left\|X_{\alpha}-X_{\beta}\right\|
$$

for any $t \in[0, T]$.

Proof. The proof follows closely the one of [9, Theorem 3.7], and hence we will only prove (3.24). Therefore, one can show as in [9, Theorem 3.7], that

$$
\Gamma(t)=\int_{\mathbb{R}} \bar{U}^{2} y_{\xi} d \xi+\|h\|_{L^{1}},
$$

satisfies

$$
\frac{d}{d t} \Gamma(t) \leq C(\Gamma(t)+1)
$$

where $C$ denotes some constant dependent on $c$ (independent of time) and the partition function $\chi$ we choose. Thus Gronwall's inequality yields

$$
\Gamma(t)+1 \leq(\Gamma(0)+1) e^{C t}
$$

where $C$ depends on $c$ and the partition function $\chi$. Moreover,

$$
\Gamma_{0} \leq\left\|\bar{U}_{0}\right\|_{L^{2}}^{2}+\left\|\bar{U}_{0}\right\|_{L^{\infty}}\left\|\bar{U}_{0}\right\|_{L^{2}}\left\|\zeta_{0, \xi}\right\|_{L^{2}}+\left\|h_{0}\right\|_{L^{1}}
$$

Hence $\Gamma(t)$ only depends on $t$, the partition function $\chi$ and $\left\|X_{0}\right\|_{E}$. Thus, using the estimates derived in [9, Theorem 3.7], one obtains that $\left\|S_{t}\left(X_{\alpha}\right)\right\|_{E}$ depends only 
on $\left\|X_{0}\right\|_{E}, t$, and the partition function $\chi$ we choose. Using that $F$ is Lipschitz continuous, we finally end up with (3.24).

\section{From Eulerian to Lagrangian coordinates and vice versa}

Definition 4.1. The set $\mathcal{D}$ is composed of all pairs $(u, \rho, \mu)$ such that $u \in H_{0, \infty}(\mathbb{R})$, $\rho \in L_{\text {const }}^{2}(\mathbb{R})$ and $\mu$ is a positive finite Radon measure whose absolutely continuous part, $\mu_{\mathrm{ac}}$, satisfies

$$
\mu_{\mathrm{ac}}=\left(u_{x}^{2}+\bar{\rho}^{2}\right) d x .
$$

Definition 4.2. We denote by $G$ the subgroup of the group of homeomorphisms from $\mathbb{R}$ to $\mathbb{R}$ such that

$$
\begin{gathered}
f-\mathrm{Id} \text { and } f^{-1}-\mathrm{Id} \text { both belong to } W^{1, \infty}(\mathbb{R}), \\
f_{\xi}-1 \text { belongs to } L^{2}(\mathbb{R}),
\end{gathered}
$$

where Id denotes the identity function. Given $\kappa>0$, we denote by $G_{\kappa}$ the subset $G_{\kappa}$ of $G$ defined by

$$
G_{\kappa}=\left\{f \in G \mid\|f-\mathrm{Id}\|_{W^{1, \infty}}+\left\|f^{-1}-\mathrm{Id}\right\|_{W^{1, \infty}} \leq \kappa\right\} .
$$

Lemma 4.3 ([18, Lemma 3.2]). Let $\kappa \geq 0$. If $f$ belongs to $G_{\kappa}$, then $1 /(1+\kappa) \leq$ $f_{\xi} \leq 1+\kappa$ almost everywhere. Conversely, if $f$ is absolutely continuous, $f-\operatorname{Id} \in$ $W^{1, \infty}(\mathbb{R}), f$ satisfies $4.2 \mathrm{~b}$ ) and there exists $d \geq 1$ such that $1 / d \leq f_{\xi} \leq d$ almost everywhere, then $f \in G_{\kappa}$ for some $\kappa$ depending only on $d$ and $\|f-\operatorname{Id}\|_{W^{1, \infty}}$.

We define the subsets $\mathcal{F}_{\kappa}$ and $\mathcal{F}$ of $\mathcal{G}$ as follows

$$
\mathcal{F}_{\kappa}=\left\{X=(y, U, h, r) \in \mathcal{G} \mid y+H \in G_{\kappa}\right\},
$$

and

$$
\mathcal{F}=\{X=(y, U, h, r) \in \mathcal{G} \mid y+H \in G\},
$$

where $H(t, \xi)$ is defined by

$$
H(t, \xi)=\int_{-\infty}^{\xi} h(t, \tau) d \tau
$$

which is finite since, from (3.23c), we have $h=U_{\xi}^{2}+\bar{r}^{2}-\zeta_{\xi} h$ and therefore $h \in L^{1}(\mathbb{R})$. For $\kappa=0$, we have $G_{0}=\{\operatorname{Id}\}$. As we shall see, the space $\mathcal{F}_{0}$ will play a special role. These sets are relevant only because they are preserved by the governing equation (3.15) as the next lemma shows. In particular, while the mapping $\xi \mapsto y(t, \xi)$ may not be a diffeomorphism for some time $t$, the mapping $\xi \mapsto y(t, \xi)+H(t, \xi)$ remains a diffeomorphism for all times $t$.

Lemma 4.4. The space $\mathcal{G}$ is preserved by the governing equations (3.15). More precisely, given $\kappa, T \geq 0$, and $X_{0} \in \mathcal{G}_{\kappa}$, we have

$$
S_{t}\left(X_{0}\right) \in \mathcal{G}_{\kappa^{\prime}}
$$

for all $t \in[0, T]$ where $\kappa^{\prime}$ only depends on $T, \kappa$, and $\left\|X_{0}\right\|_{E}$.

Proof. See [18, Lemma 3.3].

For the sake of simplicity, for any $X=(y, U, h, r) \in \mathcal{F}$ and any function $f \in G$, we denote $\left(y \circ f, U \circ f, h \circ f f_{\xi}, r \circ f f_{\xi}\right)$ by $X \circ f$.

Proposition 4.5. The map from $G \times \mathcal{F}$ to $\mathcal{F}$ given by $(f, X) \mapsto X \circ f$ defines an action of the group $G$ on $\mathcal{F}$. 
Proof. See [18, Proposition 3.4].

Since $G$ is acting on $\mathcal{F}$, we can consider the quotient space $\mathcal{F} / G$ of $\mathcal{F}$ with respect to the action of the group $G$. The equivalence relation on $\mathcal{F}$ is defined as follows: For any $X, X^{\prime} \in \mathcal{F}$, we say that $X$ and $X^{\prime}$ are equivalent if there exists $f \in G$ such that $X^{\prime}=X \circ f$. We denote by $\Pi(X)=[X]$ the projection of $\mathcal{F}$ into the quotient space $\mathcal{F} / G$, and introduce the mapping $\Gamma: \mathcal{F} \rightarrow \mathcal{F}_{0}$ given by

$$
\Gamma(X)=X \circ(y+H)^{-1}
$$

for any $X=(y, U, h, r) \in \mathcal{F}$. We have $\Gamma(X)=X$ when $X \in \mathcal{F}_{0}$. It is not hard to prove that $\Gamma$ is invariant under the $G$ action, that is, $\Gamma(X \circ f)=\Gamma(X)$ for any $X \in \mathcal{F}$ and $f \in G$. Hence, there corresponds to $\Gamma$ a mapping $\tilde{\Gamma}$ from the quotient space $\mathcal{F} / G$ to $\mathcal{F}_{0}$ given by $\tilde{\Gamma}([X])=\Gamma(X)$ where $[X] \in \mathcal{F} / G$ denotes the equivalence class of $X \in \mathcal{F}$. For any $X \in \mathcal{F}_{0}$, we have $\tilde{\Gamma} \circ \Pi(X)=\Gamma(X)=X$. Hence, $\left.\tilde{\Gamma} \circ \Pi\right|_{\mathcal{F}_{0}}=\left.\operatorname{Id}\right|_{\mathcal{F}_{0}}$. Any topology defined on $\mathcal{F}_{0}$ is naturally transported into $\mathcal{F} / G$ by this isomorphism. We equip $\mathcal{F}_{0}$ with the metric induced by the $E$-norm, i.e., $d_{\mathcal{F}_{0}}\left(X, X^{\prime}\right)=\left\|X-X^{\prime}\right\|_{E}$ for all $X, X^{\prime} \in \mathcal{F}_{0}$. Since $\mathcal{F}_{0}$ is closed in $E$, this metric is complete. We define the metric on $\mathcal{F} / G$ as

$$
d_{\mathcal{F} / G}\left([X],\left[X^{\prime}\right]\right)=\left\|\Gamma(X)-\Gamma\left(X^{\prime}\right)\right\|_{E},
$$

for any $[X],\left[X^{\prime}\right] \in \mathcal{F} / G$. Then, $\mathcal{F} / G$ is isometrically isomorphic with $\mathcal{F}_{0}$ and the metric $d_{\mathcal{F} / G}$ is complete.

Lemma 4.6. Given $\alpha \geq 0$. The restriction of $\Gamma$ to $\mathcal{F}_{\alpha}$ is a continuous mapping from $\mathcal{F}_{\alpha}$ to $\mathcal{F}_{0}$.

Proof. See [18, Lemma 3.5].

Remark 4.7. The mapping $\Gamma$ is not continuous from $\mathcal{F}$ to $\mathcal{F}_{0}$. The spaces $\mathcal{F}_{\alpha}$ were precisely introduced in order to make the mapping $\Gamma$ continuous.

We denote by $S: \mathcal{F} \times[0, \infty) \rightarrow \mathcal{F}$ the continuous semigroup which to any initial data $X_{0} \in \mathcal{F}$ associates the solution $X(t)$ of the system of differential equations (3.15) at time $t$. As indicated earlier, the two-component Camassa-Holm system is invariant with respect to relabeling. More precisely, using our terminology, we have the following result.

Theorem 4.8. For any $t>0$, the mapping $S_{t}: \mathcal{F} \rightarrow \mathcal{F}$ is $G$-equivariant, that is,

$$
S_{t}(X \circ f)=S_{t}(X) \circ f
$$

for any $X \in \mathcal{F}$ and $f \in G$. Hence, the mapping $\tilde{S}_{t}$ from $\mathcal{F} / G$ to $\mathcal{F} / G$ given by

$$
\tilde{S}_{t}([X])=\left[S_{t} X\right]
$$

is well-defined. It generates a continuous semigroup.

Proof. See [18, Theorem 3.7]. 
We have the following diagram:

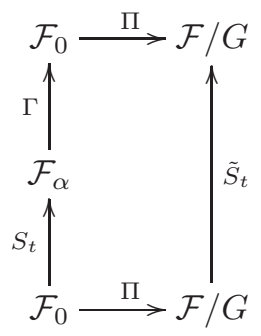

4.1. Mappings between the two coordinate systems. Our next task is to derive the correspondence between Eulerian coordinates (functions in $\mathcal{D}$ ) and Lagrangian coordinates (functions in $\mathcal{F} / G$ ). The set $\mathcal{D}$ however allows the energy density to have a singular part and a positive amount of energy can concentrate on a set of Lebesgue measure zero.

We define the mapping $L$ from $\mathcal{D}$ to $\mathcal{F}_{0}$ which to any initial data in $\mathcal{D}$ associates an initial data for the equivalent system in $\mathcal{F}_{0}$.

Theorem 4.9. For any $(u, \rho, \mu)$ in $\mathcal{D}$, let

$$
\begin{aligned}
y(\xi) & =\sup \{y \mid \mu((-\infty, y))+y<\xi\}, \\
h(\xi) & =1-y_{\xi}(\xi), \\
U(\xi) & =u \circ y(\xi), \\
r(\xi) & =\rho \circ y(\xi) y_{\xi}(\xi) .
\end{aligned}
$$

Then $(y, U, h, r) \in \mathcal{F}_{0}$. We denote by $L: \mathcal{D} \rightarrow \mathcal{F}$ the mapping which to any element $(u, \rho, \mu) \in \mathcal{D}$ associates $X=(y, U, h, r) \in \mathcal{F}$ given by (4.6).

Proof. The proof follows the same lines as the one of [18, Theorem 3.8], which we do not repeat here. We give some more details about the variable $\rho$ and its Lagrangian counterpart $r$, which are specific to the Camassa-Holm system. As in [18, Theorem $3.8]$, we obtain that

$$
\left(u_{x}^{2} \circ y+\bar{\rho}^{2} \circ y+1\right) y_{\xi}=1
$$

for allmost every $\xi$ such that $y_{\xi} \neq 0$. From (4.7), it follows that $0 \leq y_{\xi} \leq 1$ and $h y_{\xi}=\left(u_{\xi} \circ y\right)^{2} y_{\xi}^{2}+(\bar{\rho} \circ y)^{2} y_{\xi}^{2}$, which implies (3.23c). It remains to prove that $\bar{r} \in L^{2}(\mathbb{R}) \cap L^{\infty}(\mathbb{R})$. We have

$$
\int_{\mathbb{R}} \bar{r}(\xi)^{2} d \xi=\int_{\mathbb{R}} \bar{\rho}(y(\xi))^{2} y_{\xi}^{2} d \xi \leq \int_{\mathbb{R}} \bar{\rho}(y(\xi))^{2} y_{\xi} d \xi=\int_{\mathbb{R}} \bar{\rho}(x)^{2} d x<\infty
$$

and

$$
\bar{r}^{2}=\bar{\rho}^{2} \circ \text { y } y_{\xi}^{2} \leq \bar{\rho}^{2} \circ \text { y } y_{\xi} \leq 1 .
$$

Reversely, to any element in $\mathcal{F}$ there corresponds a unique element in $\mathcal{D}$ which is given by the mapping $M$ defined below.

Theorem 4.10. Given any element $X=(y, U, h, r) \in \mathcal{F}$. Then, the measure $y_{\#}(\bar{r}(\xi) d \xi)$ is absolutely continuous, and we define $(u, \rho, \mu)$ as follows

$$
\begin{aligned}
& u(x)=U(\xi) \text { for any } \xi \text { such that } x=y(\xi), \\
& \mu=y_{\#}(h(\xi) d \xi)
\end{aligned}
$$




$$
\begin{aligned}
& \bar{\rho}(x) d x=y_{\#}(\bar{r}(\xi) d \xi), \\
& \rho(x)=k+\bar{\rho}(x) .
\end{aligned}
$$

We have that $(u, \rho, \mu)$ belongs to $\mathcal{D}$. We denote by $M: \mathcal{F} \rightarrow \mathcal{D}$ the mapping which to any $X$ in $\mathcal{F}$ associates the element $(u, \rho, \mu) \in \mathcal{D}$ as given by (4.8). In particular, the mapping $M$ is invariant under relabeling.

Proof. Most of the proof follows closely [18, Theorem 3.11]. That the definition of $u$ is well-posed follows exactly as in [18, Theorem 3.11]. Next we prove that $y_{\#}(\bar{r}(\xi) d \xi)$ is absolutely continuous and satisfies (4.8C) for $\bar{\rho} \in L^{2}(\mathbb{R})$. In addition, we will see that $\bar{\rho} \circ y y_{\xi}=\bar{r}$ whenever $y_{\xi} \neq 0$. Let $\lambda=y_{\#}(\bar{r}(\xi) d \xi)$. For any continuous function with compact support $\phi$, we have, using the change of variables $x=y(\xi)$,

$$
\int_{\mathbb{R}} \phi(x) d \lambda(x)=\int_{\mathbb{R}} \bar{r} \phi \circ y d \xi=\int_{\left\{\xi \in \mathbb{R} \mid y_{\xi}(\xi) \neq 0\right\}} \frac{\bar{r}}{\sqrt{y_{\xi}}} \phi \circ y \sqrt{y_{\xi}} d \xi,
$$

because $\bar{r}(\xi)=0$ for almost every $\xi$ such that $y_{\xi}=0$. Hence,

$$
\begin{aligned}
\int_{\mathbb{R}} \phi(x) d \lambda(x) & \leq\left(\int_{\left\{\xi \in \mathbb{R} \mid y_{\xi}(\xi) \neq 0\right\}} \frac{\bar{r}^{2}}{y_{\xi}} d \xi\right)^{1 / 2}\left(\int_{\mathbb{R}} \phi^{2} d x\right)^{1 / 2} \\
& \leq\left(\int_{\mathbb{R}} h d \xi\right)^{1 / 2}\|\phi\|_{L^{2}} \leq\|h\|_{L^{1}}^{1 / 2}\|\phi\|_{L^{2}},
\end{aligned}
$$

where we used that $\bar{r}^{2} \leq h y_{\xi}$, see (3.23c). It follows that $\lambda$ is absolutely continuous, we write $\lambda=\bar{\rho} d x$, and (4.9) also implies that $\bar{\rho} \in L^{2}(\mathbb{R})$ with $\|\bar{\rho}\|_{L^{2}} \leq\|h\|_{L^{1}}^{\frac{1}{2}}$. By the definition of $\bar{\rho}$, we have that, for any set $B$,

$$
\int_{B} \bar{\rho}(x) d x=\int_{y^{-1}(B)} \bar{\rho} \circ y y_{\xi} d \xi=\int_{y^{-1}(B)} \bar{r} d \xi
$$

Define

$$
\begin{gathered}
Z=\left\{\xi \in \mathbb{R} \mid y \text { is differentiable at } \xi \text { and } y_{\xi}(\xi)=0\right. \\
\text { or } y \text { is not differentiable at } \xi\},
\end{gathered}
$$

For any $\tilde{B} \subset Z^{c}$, we have $y^{-1}(y(\tilde{B}))=\tilde{B}$. Indeed, assume the opposite. Then, there exists $\xi_{0} \in \tilde{B}$ and $\xi_{1} \in \tilde{B}^{c}$ such that $y\left(\xi_{0}\right)=y\left(\xi_{1}\right)$. Since $y$ is increasing, it implies that $y_{\xi}\left(\xi_{0}\right)=0$, which contradicts the fact that $\xi_{0} \in Z^{c}$. Thus, (4.10) gives

$$
\int_{\tilde{B}} \bar{\rho} \circ y y_{\xi} d \xi=\int_{\tilde{B}} \bar{r} d \xi
$$

and it follows that

$$
\bar{\rho} \circ y y_{\xi}=\bar{r}
$$

on $Z^{c}$. Finally, it is left to show that $\mu_{a c}=u_{x}^{2}+\bar{\rho}^{2}$. We can use (4.11) and the proof follows the same line as in [18, Theorem 3.11].

Finally, in order to show that the equivalence classes in Lagrangian coordinates are in bijection with the set of Eulerian coordinates, it is left to prove the following theorem.

Theorem 4.11. The mappings $M$ and $L$ are invertible. We have

$$
L \circ M=\operatorname{Id}_{\mathcal{F} / G} \text { and } M \circ L=\operatorname{Id}_{\mathcal{D}} \text {. }
$$


Proof. Given $[X]$ in $\mathcal{F} / G$, we choose $X=(y, U, h, r)=\tilde{\Gamma}([X])$ as a representative of $[X]$ and consider $(u, \rho, \mu)$ given by (4.8) for this particular $X$. Note that, from the definition of $\tilde{\Gamma}$, we have $X \in \mathcal{F}_{0}$. Let $\tilde{X}=(\tilde{y}, \tilde{U}, \tilde{h}, \tilde{r})$ be the representative of $L(u, \rho, \mu)$ in $\mathcal{F}_{0}$ given by the formulas (4.6). We claim that $(\tilde{y}, \tilde{U}, \tilde{h}, \tilde{r})=(y, U, h, r)$ and therefore $L \circ M=\operatorname{Id}_{\mathcal{F} / G}$. Let

$$
g(x)=\sup \{\xi \in \mathbb{R} \mid y(\xi)<x\} .
$$

It is not hard to prove, using the fact that $y$ is increasing and continuous, that

$$
y(g(x))=x
$$

and $y^{-1}((-\infty, x))=(-\infty, g(x))$. For any $x \in \mathbb{R}$, we have, by (4.8b), that

$$
\mu((-\infty, x))=\int_{y^{-1}((-\infty, x))} h d \xi=\int_{-\infty}^{g(x)} h d \xi=H(g(x))
$$

because $H(-\infty)=0$. Since $X \in \mathcal{F}_{0}, y+H=\mathrm{Id}$ and we get

$$
\mu((-\infty, x))+x=g(x) .
$$

From the definition of $\tilde{y}$, we then obtain that

$$
\tilde{y}(\xi)=\sup \{x \in \mathbb{R} \mid g(x)<\xi\} .
$$

For any given $\xi \in \mathbb{R}$, let us consider an increasing sequence $x_{i}$ tending to $\tilde{y}(\xi)$ such that $g\left(x_{i}\right)<\xi$; such sequence exists by (4.15). Since $y$ is increasing and using (4.13), it follows that $x_{i} \leq y(\xi)$. Letting $i$ tend to $\infty$, we obtain $\tilde{y}(\xi) \leq y(\xi)$. Assume that $\tilde{y}(\xi)<y(\xi)$. Then, there exists $x$ such that $\tilde{y}(\xi)<x<y(\xi)$ and equation (4.15) then implies that $g(x) \geq \xi$. On the other hand, $x=y(g(x))<y(\xi)$ implies $g(x)<\xi$ because $y$ is increasing, which gives us a contradiction. Hence, we have $\tilde{y}=y$. It follows directly from the definitions, since $y_{\xi}+h=1$, that $\tilde{h}=h$, $\tilde{U}=U,|\overline{\tilde{r}}|=|\bar{r}|$, and $\tilde{d}=d$. If $y_{\xi}(\xi)=0$, then $\overline{\tilde{r}}(\xi)=0=\bar{r}(\xi)$. We use (4.11) and get $\overline{\tilde{r}}(\xi)=\bar{\rho}(\tilde{y}(\xi)) \tilde{y}_{\xi}=\bar{\rho}(y(\xi)) y_{\xi}=\bar{r}$ if $y_{\xi}(\xi) \neq 0$. Thus we have proved that $L \circ M=\operatorname{Id}_{\mathcal{F} / G}$.

We now turn to the proof that $M \circ L=\operatorname{Id}_{\mathcal{D}}$. Given $(u, \rho, \mu)$ in $\mathcal{D}$, we denote by $(y, U, h, r)$ the representative of $L(u, \rho, \mu)$ in $\mathcal{F}_{0}$ given by (4.6). Then, let $(\tilde{u}, \tilde{\rho}, \tilde{\mu})=$ $M \circ L(u, \rho, \mu)$. We claim that $(\tilde{u}, \tilde{\rho}, \tilde{\mu})=(u, \rho, \mu)$. Let $g$ be the function defined as before by (4.12). The same computation that leads to (4.14) now gives

$$
\tilde{\mu}((-\infty, x))+x=g(x) .
$$

Given $\xi \in \mathbb{R}$, we consider an increasing sequence $x_{i}$ which converges to $y(\xi)$ and such that $\mu\left(\left(-\infty, x_{i}\right)\right)+x_{i}<\xi$. The existence of such a sequence is guaranteed by (4.6a). Passing to the limit and since $F(x)=\mu((-\infty, x))$ is lower semi-continuous, we obtain $\mu((-\infty, y(\xi)))+y(\xi) \leq \xi$. We take $\xi=g(x)$ and get

$$
\mu((-\infty, x))+x \leq g(x) .
$$

From the definition of $g$, there exists an increasing sequence $\xi_{i}$ which converges to $g(x)$ such that $y\left(\xi_{i}\right)<x$. The definition (4.6a) of $y$ tells us that $\mu((-\infty, x))+x \geq \xi_{i}$. Letting $i$ tend to infinity, we obtain $\mu((-\infty, x))+x \geq g(x)$ which, together with (4.17), yields

$$
\mu((-\infty, x))+x=g(x) .
$$


K. GRUNERT, H. HOLDEN, AND X. RAYNAUD

Comparing (4.18) and (4.16) we get that $\mu=\tilde{\mu}$. It is clear from the definitions that $\tilde{u}=u$. Using (4.11), we get $\tilde{\rho}(y) y_{\xi}=r=\rho(y) y_{\xi}$ and therefore $\tilde{\rho}=\rho$. Hence, $(\tilde{u}, \tilde{\rho}, \tilde{\mu})=(u, \rho, \mu)$ and $M \circ L=\operatorname{Id}_{\mathcal{D}}$.

\section{Continuous Semigroup of SOlutions}

We define $T_{t}$ as

$$
T_{t}=M \circ S_{t} \circ L .
$$

The metric $d_{\mathcal{D}}$ is defined as

$$
d_{D}\left(\left(u_{1}, \rho_{1}, \mu_{1}\right),\left(u_{2}, \rho_{2}, \mu_{2}\right)\right)=d_{\mathcal{F}_{0}}\left(L\left(u_{1}, \rho_{1}, \mu_{1}\right), L\left(u_{2}, \rho_{2}, \mu_{2}\right)\right) .
$$

Definition 5.1. Assume that $u:[0, \infty) \times \mathbb{R} \rightarrow \mathbb{R}$ and $\rho:[0, \infty) \times \mathbb{R} \rightarrow \mathbb{R}$ satisfy (i) $u \in L_{\mathrm{loc}}^{\infty}\left([0, \infty), H_{\infty}(\mathbb{R})\right)$ and $\rho \in L_{\mathrm{loc}}^{\infty}\left([0, \infty), L_{\text {const }}^{2}(\mathbb{R})\right)$,

(ii) the equations

$$
\begin{array}{r}
\iint_{[0, \infty) \times \mathbb{R}}\left[-u(t, x) \phi_{t}(t, x)+\left(u(t, x) u_{x}(t, x)+P_{x}(t, x)\right) \phi(t, x)\right] d x d t \\
=\int_{\mathbb{R}} u(0, x) \phi(0, x) d x
\end{array}
$$

$$
\iint_{[0, \infty) \times \mathbb{R}}\left[\left(P(t, x)-u^{2}(t, x)-\frac{1}{2} u_{x}^{2}(t, x)-\frac{1}{2} \rho^{2}(t, x)\right) \phi(t, x)+P_{x}(t, x) \phi_{x}(t, x)\right] d x d t=0,
$$

and

$$
\iint_{[0, \infty) \times \mathbb{R}}\left[-\rho(t, x) \phi_{t}(t, x)-u(t, x) \rho(t, x) \phi_{x}(t, x)\right] d x d t=\int_{\mathbb{R}} \rho(0, x) \phi(0, x) d x,
$$

hold for all $\phi \in C_{0}^{\infty}([0, \infty) \times \mathbb{R})$. Then we say that $u$ is a weak global solution of the two-component Camassa-Holm system. If $u$ in addition satisfies

$$
\left(u^{2}+u_{x}^{2}+\rho^{2}\right)_{t}+\left(u\left(u^{2}+u_{x}^{2}+\rho^{2}\right)\right)_{x}-\left(u^{3}-2 P u\right)_{x}=0
$$

in the sense that

$$
\begin{aligned}
\iint_{(0, \infty) \times \mathbb{R}}\left[\left(u^{2}(t, x)+u_{x}^{2}(t, x)+\rho^{2}(t, x)\right) \phi_{t}(t, x)\right. \\
+\left(u(t, x)\left(u^{2}(t, x)+u_{x}^{2}(t, x)+\rho^{2}(t, x)\right)\right) \phi_{x}(t, x) \\
\left.-\left(u^{3}(t, x)-2 P(t, x) u(t, x)\right) \phi_{x}(t, x)\right] d x d t=0,
\end{aligned}
$$

for any $\phi \in C_{0}^{\infty}((0, \infty) \times \mathbb{R})$, we say that $u$ is a weak global conservative solution of the two-component Camassa-Holm system.

Theorem 5.2. The mapping $T_{t}$ is a continuous semigroup of solutions with respect to the metric $d_{\mathcal{D}}$. Given any initial data $\left(u_{0}, \rho_{0}, \mu_{0}\right) \in \mathcal{D}$, let $\left.\left.(u(t, \cdot), \rho(t, \cdot)), \mu(t, \cdot)\right)\right)=$ $T_{t}\left(u_{0}, \rho_{0}, \mu_{0}\right)$. Then $(u, \rho)$ is a weak solution to (1.1), and $\mu$ is a weak solution to

$$
\left(u^{2}+\mu+\rho^{2}-\bar{\rho}^{2}\right)_{t}+\left(u\left(u^{2}+\mu+\rho^{2}-\bar{\rho}^{2}\right)\right)_{x}=\left(u^{3}-2 P u\right)_{x} .
$$

For almost every time, $\mu$ is absolutely continuous, and in that case $\mu=\left(u_{x}^{2}+\bar{\rho}^{2}\right) d x$.

Proof. The proof follows closely the one for the Camassa-Holm equation with nonvanishing asymptotics, and we therefore refer to [9, Theorem 5.2]. 


\section{Regularity and uniqueness Results}

Given $(u, \rho, \mu) \in \mathcal{D}, p \in \mathbb{N}$ and an open set $I$, we say that $(u, \rho, \mu)$ is $p$-regular on an open set $I$ if

$$
u \in W^{p, \infty}(I), \rho \in W^{p-1, \infty}(I) \text { and } \mu_{\mathrm{ac}}=\mu \text { on } I .
$$

By notation, we set $W^{0, \infty}(I)=L^{\infty}(I)$. The variable $\rho$ has a regularizing effect, as the following theorem shows. Even if the $2 \mathrm{CH}$ system has an infinite speed of propagation, see [17, we obtain the hyperbolic feature that discontinuities travel at finite speed. The regularity is preserved in intervals defined by the characteristics.

Theorem 6.1. We consider the initial data $\left(u_{0}, \rho_{0}, \mu_{0}\right)$. Assume that $\left(u_{0}, \rho_{0}, \mu_{0}\right)$ is p-regular on a given interval $\left(x_{0}, x_{1}\right)$ and

$$
\rho_{0}(x)^{2} \geq c>0
$$

for $x \in\left(x_{0}, x_{1}\right)$. Then, for any $t \in \mathbb{R}_{+},(u, \rho, \mu)(t, \cdot)$ is p-regular on the interval $\left(y\left(t, \xi_{0}\right), y\left(t, \xi_{1}\right)\right)$, where $\xi_{0}$ and $\xi_{1}$ satisfy $y\left(0, \xi_{0}\right)=x_{0}$ and $y\left(0, \xi_{1}\right)=x_{1}$ and are defined as

$$
\xi_{0}=\sup \left\{\xi \in \mathbb{R} \mid y(0, \xi) \leq x_{0}\right\} \text { and } \xi_{1}=\inf \left\{\xi \in \mathbb{R} \mid y(0, \xi) \geq x_{1}\right\} .
$$

Proof. We consider first the case $p=1$. Let $\left(y_{0}, U_{0}, h_{0}, r_{0}\right)=L\left(u_{0}, \mu_{0}, \rho_{0}\right)$. Since $y_{0}$ is surjective and continuous, we have $y_{0}\left(\xi_{0}\right)=x_{0}$ and $y_{0}\left(\xi_{1}\right)=x_{1}$. We denote $I=\left(x_{0}, x_{1}\right)$ and $J=\left(\xi_{0}, \xi_{1}\right)$. Since $\mu$ is absolutely continuous on $I$, we get from (4.6a) that, for any $\xi \in J$,

$$
\mu\left(\left(-\infty, y_{0}\left(\xi_{0}\right)\right]\right)+\mu_{\mathrm{ac}}\left(\left(y_{0}\left(\xi_{0}\right), y_{0}(\xi)\right)\right)+y_{0}(\xi)=\xi
$$

so that

$$
\mu\left(\left(-\infty, y_{0}\left(\xi_{0}\right)\right]\right)+\int_{y_{0}\left(\xi_{0}\right)}^{y_{0}(\xi)}\left(u_{0 x}^{2}+\bar{\rho}_{0}^{2}\right) d x+y_{0}(\xi)=\xi
$$

We differentiate this relation with respect to $\xi$ and obtain that

$$
y_{0 \xi}(\xi)=\frac{1}{\left(1+u_{0 x}^{2}+\bar{\rho}_{0}^{2}\right) \circ y_{0}(\xi)} .
$$

Hence,

$$
y_{0 \xi}(\xi) \geq \frac{1}{1+C}
$$

for any $\xi \in J$ and for a constant $C$ which depends only on $\left\|u_{0}\right\|_{W^{1, \infty}}(I)$ and $\left\|\rho_{0}\right\|_{L^{\infty}(I)}$, both quantities being bounded when $\left(u_{0}, \rho_{0}, \mu_{0}\right)$ is $p$-regular in $I$. By the definition of $r_{0}$, we have $\rho_{0}\left(y_{0}(\xi)\right) y_{0 \xi}(\xi)=r_{0}(\xi)$ and, therefore, it follows from (6.3) and assumption (6.1) that, for any $\xi \in J, r_{0}(\xi) \geq c_{1}$ for some constant $c_{1}>0$. By (3.20d and (3.23c), we have

$$
c_{1}^{2} \leq r_{0}^{2}(\xi)=r^{2}(t, \xi)=\left(\bar{r}+k y_{\xi}\right)^{2}=\bar{r}^{2}+y_{\xi}\left(2 \bar{r}+y_{\xi}\right) \leq y_{\xi}\left(h+2 \bar{r}+y_{\xi}\right)
$$

for any $t \in[0, T]$ and $\xi \in J$. In Lemma 3.5, it is shown that

$$
\|\zeta(t, \cdot)\|_{W^{1, \infty}}+\|U(t, \cdot)\|_{W^{1, \infty}}+\|h(t, \cdot)\|_{L^{\infty}}+\|r(t, \cdot)\|_{L^{\infty}} \leq C_{1}
$$

for some constant $C_{1}$ which depends on the initial data. Then, (6.4) yields $y_{\xi}(t, \xi) \geq$ $c_{2}$ for some constant $c_{2}>0$. It follows that for each $t \in[0, T]$, the mapping $y(t, \cdot):\left(\xi_{0}, \xi_{1}\right) \mapsto\left(y\left(t, \xi_{0}\right), y\left(t, \xi_{1}\right)\right)$ is a Lipschitz homeomorphism and its inverse 
$y^{-1}$ is also Lipschitz. We denote the interval $\left(y\left(t, \xi_{0}\right), y\left(t, \xi_{1}\right)\right)$ by $I^{t}$. By the definitions of Theorem 4.10, it follows that

$$
u(t, x)=U\left(t, y^{-1}(t, x)\right) \text { and } \rho(t, x)=\frac{r\left(t, y^{-1}(t, x)\right)}{y_{\xi}\left(t, y^{-1}(t, x)\right)}
$$

for $x \in I^{t}$. Hence, $u(t, \cdot) \in W^{1, \infty}\left(I^{t}\right)$ and $\rho(t, \cdot) \in L^{\infty}\left(I^{t}\right)$. Since $\mu=y_{\#}(h(\xi) d \xi)$ and $y^{-1}$ is Lipschitz on $I^{t}$, we have

$$
\mu(t, A)=\int_{y^{-1}(A)} h(t, \xi) d \xi=\int_{A} \frac{h \circ y^{-1}}{y_{\xi} \circ y^{-1}} d x=\int_{A}\left(u_{x}^{2}(t, x)+\bar{\rho}(t, x)^{2}\right) d x
$$

for any subset $A$ of $I^{t}$. Hence, $\mu=\mu_{\text {ac }}$ in $I^{t}$. Let us now consider the case $p>1$. It follows from (6.2) that $y_{0} \in W^{p, \infty}(J)$. Since $U_{0}(\xi)=u_{0} \circ y_{0}(\xi)$ on $J$, we get $U_{0} \in W^{p, \infty}(J)$. Similarly, (4.6b) and 4.6d yield $h_{0} \in W^{p-1, \infty}(J)$ and $r_{0} \in W^{p-1, \infty}(J)$. The key point is that, due to the quasilinear structure of the equivalent system (3.20), this regularity is preserved by the flow. By differentiating $Q$ given by (3.13), we observe that $\partial_{\xi}^{p} Q$ is quasilinear in $\partial_{\xi}^{p} y, \partial_{\xi}^{p} U, \partial_{\xi}^{p-1} h$ and $\partial_{\xi}^{p-1} \bar{r}$, that is, it can be written as

$$
\partial_{\xi}^{p} Q_{\xi}=a_{1} \partial_{\xi}^{p} y_{\xi}+a_{2} \partial_{\xi}^{p} U_{\xi}+a_{3} \partial_{\xi}^{p-1} h+a_{4} \partial_{\xi}^{p-1} \bar{r}+a_{5}
$$

where $\left\{a_{i}\right\}_{i=1}^{5}$ are functions that are bounded in $L^{\infty}(J)$ by a constant depending only on $\|y-\operatorname{Id}\|_{W^{p-1, \infty}(J)},\|U\|_{W^{p-1, \infty}(J)},\|h\|_{W^{p-2, \infty}(J)},\|\bar{r}\|_{W^{p-2, \infty}(J)},\|P\|_{L^{\infty}(J)}$ and $\|Q\|_{L^{\infty}(J)}$. The same property holds for $P$. One checks directly that the system (3.20) inherits the same quasilinearity property, that is, the $p$ th derivative of each term on the right-hand side can be written as a linear combination of $\partial_{\xi}^{p} y, \partial_{\xi}^{p} U$, $\partial_{\xi}^{p-1} h$ and $\partial_{\xi}^{p-1} \bar{r}$ as in (6.6). Then, we use Gronwall's lemma and an induction argument on $j=1, \ldots, p$ to prove, that if

$$
(\zeta(t, \cdot), U(t, \cdot), h(t, \cdot), r(t, \cdot)) \in W^{j, \infty}(J) \times W^{j, \infty}(J) \times W^{j-1, \infty}(J) \times W^{j-1, \infty}(J)
$$

holds for $t=0$, then it remains true for $t \in[0, T]$, for $j=1, \ldots, p$. Using (6.5), we conclude that $u \in W^{p, \infty}\left(I^{t}\right)$ and $\rho \in W^{p-1, \infty}\left(I^{t}\right)$.

Corollary 6.2. If the initial data $\left(u_{0}, \rho_{0}, \mu_{0}\right) \in \mathcal{D}$ satisfies $u_{0}, \rho_{0} \in C^{\infty}(\mathbb{R}), \mu_{0}$ is absolutely continuous and $\rho_{0}^{2}(x) \geq d>0$ for all $x \in \mathbb{R}$, then $u, \rho \in C^{\infty}(\mathbb{R} \times \mathbb{R})$ is the unique classical solution to (3.1).

These classical solutions can be used to obtain the global conservative solution of the Camassa-Holm equation (1.4). We consider only initial data for which $\mu_{0}$ is absolutely continuous.

Theorem 6.3. Let $u_{0} \in H_{\infty}(\mathbb{R})$. We consider the approximating sequence of initial data $\left(u_{0}^{n}, \rho_{0}^{n}, \mu_{0}^{n}\right) \in \mathcal{D}$ given by $u_{0}^{n} \in C^{\infty}(\mathbb{R})$ with $\lim _{n \rightarrow \infty} u_{0}^{n}=u_{0}$ in $H_{\infty}(\mathbb{R})$, $\rho_{0}^{n} \in C^{\infty}(\mathbb{R})$ with $\lim _{n \rightarrow \infty} \rho_{0}^{n}=0$ in $L_{\text {const }}^{2}(\mathbb{R}),\left(\rho_{0}^{n}\right)^{2} \geq d_{n}$ for some constant $d_{n}>0$ and for all $n$ and $\mu_{0}^{n}=\left(\left(u_{0, x}^{n}\right)^{2}+\left(\bar{\rho}_{0}^{n}\right)^{2}\right) d x$. We denote by $\left(u^{n}, \rho^{n}\right)$ the unique classical solution to (3.1) in $C^{\infty}\left(\mathbb{R}_{+} \times \mathbb{R}\right) \times C^{\infty}\left(\mathbb{R}_{+} \times \mathbb{R}\right)$ which corresponds to this initial data. Then for every $t \in \mathbb{R}_{+}$, the sequence $u^{n}(t, \cdot)$ converges to $u(t, \cdot)$ in $L^{\infty}(\mathbb{R})$, where $u$ is the conservative solution of the Camassa-Holm equation (1.4) with initial data $u_{0} \in H_{\infty}(\mathbb{R})$. 
Proof. The proof relies on the stability of the semigroup of solutions and on the Lemmas 6.4 and 6.5 below, which compare the topology of $\mathcal{D}$ with standard topologies. From Lemma 6.4 it follows that $\left(u_{0}^{n}, \rho_{0}^{n}, \mu_{0}^{n}\right)$ converges to $\left(u_{0}, 0, \mu_{0}\right)$ in $\mathcal{D}$. By the stability of the semigroup with respect to $\mathcal{D}$, we have that, for any $t \in \mathbb{R}_{+}$, $\left(u^{n}(t), \rho^{n}(t), \mu^{n}(t)\right)$ converges to $(u(t), 0, \mu(t))$ in $\mathcal{D}$. Then, Lemma 6.5 gives that $u^{n}(t, \cdot)$ converges to $u(t, \cdot)$ in $L^{\infty}(\mathbb{R})$.

In order to complete the proof of the previous and main theorem, we show the following lemmas.

Lemma 6.4. The mapping

$$
(u, \rho) \mapsto\left(u, \rho,\left(u_{x}^{2}+\bar{\rho}^{2}\right) d x\right)
$$

is continuous from $H_{0, \infty}(\mathbb{R}) \times L_{\text {const }}^{2}(\mathbb{R})$ into $\mathcal{D}$. In other words, given a sequence $\left(u_{n}, \rho_{n}\right)$ in $H_{0, \infty}(\mathbb{R}) \times L_{\text {const }}^{2}(\mathbb{R})$ which converges to $(u, \rho)$ in $H_{0, \infty}(\mathbb{R}) \times L_{\text {const }}^{2}(\mathbb{R})$, then $\left(u_{n}, \rho_{n},\left(u_{n, x}^{2}+\bar{\rho}_{n}^{2}\right) d x\right)$ converges to $\left(u, \rho,\left(u_{x}^{2}+\bar{\rho}^{2}\right) d x\right)$ in $\mathcal{D}$.

Proof. The proof follows the same lines as the one of [18, Proposition 5.1], which we will not repeat here. Here we focus on showing that $\bar{r}_{n} \rightarrow \bar{r} \in L^{2}(\mathbb{R})$. Note that we know already from the convergence in Eulerian coordinates that $\left|k_{n}-k\right|$ converges to 0 .

We write $g_{n}=u_{n, x}^{2}+\bar{\rho}_{n}^{2}$ and $g=u_{x}^{2}+\bar{\rho}^{2}$. Let $X_{n}=\left(y_{n}, U_{n}, h_{n}, r_{n}\right)$ and $X=(y, U, h, r)$ be the representatives in $\mathcal{F}_{0}$ given by (4.6) of $L\left(u_{n}, \rho_{n},\left(u_{n, x}^{2}+\bar{\rho}^{2}\right) d x\right)$ and $L\left(u, \rho,\left(u_{x}^{2}+\bar{\rho}^{2}\right) d x\right)$, respectively. In particular, we have

$$
\int_{-\infty}^{y(\xi)} g(x) d x+y(\xi)=\xi, \int_{-\infty}^{y_{n}(\xi)} g_{n}(x) d x+y_{n}(\xi)=\xi
$$

and, after taking the difference between the two equations, we obtain

$$
\int_{-\infty}^{y(\xi)}\left(g-g_{n}\right)(x) d x+\int_{y_{n}(\xi)}^{y(\xi)} g_{n}(x) d x+y(\xi)-y_{n}(\xi)=0 .
$$

Since $g_{n}$ is positive, $\left|y-y_{n}+\int_{y_{n}}^{y} g_{n}(x) d \xi\right|=\left|y-y_{n}\right|+\left|\int_{y_{n}}^{y} g_{n}(x) d \xi\right|$ and (6.9) implies

$$
\left|y(\xi)-y_{n}(\xi)\right| \leq \int_{-\infty}^{y(\xi)}\left|g-g_{n}\right|(x) d x \leq\left\|g-g_{n}\right\|_{L^{1}} .
$$

Since $u_{n} \rightarrow u$ in $H_{0, \infty}(\mathbb{R})$ and $\rho_{n} \rightarrow \rho \in L_{\text {const }}^{2}(\mathbb{R})$, also $g_{n} \rightarrow g$ in $L^{1}(\mathbb{R})$ and it follows that $\zeta_{n} \rightarrow \zeta \in L^{\infty}(\mathbb{R})$. The measures $\left(u_{x}^{2}+\bar{\rho}^{2}\right) d x$ and $\left(u_{n, x}^{2}+\bar{\rho}_{n}^{2}\right) d x$ have, by definition, no singular part and therefore

$$
y_{\xi}=\frac{1}{g \circ y+1} \text { and } y_{n, \xi}=\frac{1}{g_{n} \circ y_{n}+1}
$$

almost everywhere. Hence,

$$
\begin{aligned}
\zeta_{n, \xi}-\zeta_{\xi} & =\left(g \circ y-g_{n} \circ y_{n}\right) y_{n, \xi} y_{\xi} \\
& =\left(g \circ y-g \circ y_{n}\right) y_{n, \xi} y_{\xi}+\left(g \circ y_{n}-g_{n} \circ y_{n}\right) y_{n, \xi} y_{\xi} .
\end{aligned}
$$

Since $0 \leq y_{\xi} \leq 1$, we have

$$
\int_{\mathbb{R}}\left|g \circ y_{n}-g_{n} \circ y_{n}\right| y_{n, \xi} y_{\xi} d \xi \leq \int_{\mathbb{R}}\left|g \circ y_{n}-g_{n} \circ y_{n}\right| y_{n, \xi} d \xi=\left\|g-g_{n}\right\|_{L^{1}} .
$$


For any $\varepsilon>0$, there exists a continuous function $l$ with compact support such that $\|g-l\|_{L^{1}} \leq \varepsilon / 3$. We can decompose the first term on the right-hand side of (6.11) into

$$
\begin{aligned}
\left(g \circ y-g \circ y_{n}\right) y_{n, \xi} y_{\xi}= & (g \circ y-l \circ y) y_{n, \xi} y_{\xi} \\
& +\left(l \circ y-l \circ y_{n}\right) y_{n, \xi} y_{\xi}+\left(l \circ y_{n}-g \circ y_{n}\right) y_{n, \xi} y_{\xi} .
\end{aligned}
$$

Then, we have

$$
\int_{\mathbb{R}}|g \circ y-l \circ y| y_{n, \xi} y_{\xi} d \xi \leq \int|g \circ y-l \circ y| y_{\xi} d \xi=\|g-l\|_{L^{1}} \leq \frac{\varepsilon}{3},
$$

and, similarly, we obtain $\int_{\mathbb{R}}\left|g \circ y_{n}-l \circ y_{n}\right| y_{n, \xi} y_{\xi} d \xi \leq \varepsilon / 3$. Since $y_{n} \rightarrow y$ in $L^{\infty}(\mathbb{R})$ and $l$ is continuous with compact support, we obtain by applying the Lebesgue dominated convergence theorem, that $l \circ y_{n} \rightarrow l \circ y$ in $L^{1}(\mathbb{R})$, and thus we can choose $n$ big enough so that

$$
\int_{\mathbb{R}}\left|l \circ y-l \circ y_{n}\right| y_{n, \xi} y_{\xi} d \xi \leq\left\|l \circ y-l \circ y_{n}\right\|_{L^{1}} \leq \frac{\varepsilon}{3} .
$$

Hence, from (6.13), we get that $\int_{\mathbb{R}}\left|g \circ y-g \circ y_{n}\right| y_{n, \xi} y_{\xi} d \xi \leq \varepsilon$ so that

$$
\lim _{n \rightarrow \infty} \int_{\mathbb{R}}\left|g \circ y-g \circ y_{n}\right| y_{n, \xi} y_{\xi} d \xi=0,
$$

and, from (6.11) and (6.12), it follows that $\zeta_{n, \xi} \rightarrow \zeta_{\xi}$ in $L^{1}(\mathbb{R})$. Since $X_{n} \in \mathcal{F}_{0}$, $\zeta_{n, \xi}$ is bounded in $L^{\infty}(\mathbb{R})$, we finally get that $\zeta_{n, \xi} \rightarrow \zeta_{\xi}$ in $L^{2}(\mathbb{R})$ and, by (4.6b), $h_{n} \rightarrow h$ in $L^{2}(\mathbb{R})$.

We are now ready to show that $\bar{r}_{n} \rightarrow \bar{r}$ in $L^{2}(\mathbb{R})$. By definition we have $\bar{r}_{n}=$ $\bar{\rho}_{n} \circ y_{n} y_{n, \xi}$ and $\bar{r}=\bar{\rho} \circ y y_{\xi}$, so that

$$
\begin{aligned}
\left\|\bar{r}_{n}-\bar{r}\right\|_{L^{2}}^{2}= & \left\|\bar{\rho}_{n} \circ y_{n} y_{n, \xi}-\bar{\rho} \circ y y_{\xi}\right\|_{L^{2}}^{2} \\
= & \int_{\mathbb{R}}\left(\bar{\rho}_{n} \circ y_{n}\right)^{2} y_{n, \xi}\left(y_{n, \xi}-y_{\xi}\right) d \xi+\int_{\mathbb{R}} \bar{\rho}_{n} \circ y_{n} y_{n, \xi}\left(\bar{\rho}_{n} \circ y_{n}-\bar{\rho}_{n} \circ y\right) y_{\xi} d \xi \\
& +\int_{\mathbb{R}} \bar{\rho} \circ y_{n} y_{n, \xi}\left(\bar{\rho}_{n} \circ y-\bar{\rho} \circ y\right) y_{\xi} d \xi+\int_{\mathbb{R}}(\bar{\rho} \circ y)^{2} y_{\xi}\left(y_{\xi}-y_{n, \xi}\right) d \xi \\
& +\int_{\mathbb{R}} \bar{\rho} \circ y y_{\xi}\left(\bar{\rho} \circ y-\bar{\rho} \circ y_{n}\right) y_{n, \xi} d \xi+\int_{\mathbb{R}} \bar{\rho} \circ y y_{\xi}\left(\bar{\rho} \circ y_{n}-\bar{\rho}_{n} \circ y_{n}\right) y_{n, \xi} d \xi .
\end{aligned}
$$

The first and the fourth term have the same structure, and we therefore only treat the first one. Hence

$$
\left\|\left(\bar{\rho}_{n} \circ y_{n}\right)^{2} y_{n, \xi}\left(y_{n, \xi}-y_{\xi}\right)\right\|_{L^{1}} \leq\left\|y_{\xi}-y_{n, \xi}\right\|_{L^{1}}
$$

because $\left(\bar{\rho}_{n} \circ y_{n}\right)^{2} y_{n, \xi} \leq h \leq 1$ and thus it tends to 0 as $n \rightarrow \infty$. In order to investigate the fifth term we will use that $\bar{\rho} \in L^{2}(\mathbb{R})$ and therefore for any $\varepsilon>0$ there exists a continuous function $\tilde{l}$ with compact support such that $\|\bar{\rho}-\tilde{l}\|_{L^{2}} \leq$ $\varepsilon /\left(3\|\rho\|_{L^{2}}\right)$. Thus we can write

$$
\begin{aligned}
\left\|\bar{\rho} \circ y y_{\xi}\left(\bar{\rho} \circ y-\bar{\rho} \circ y_{n}\right) y_{n, \xi}\right\|_{L^{1}} \leq & \left\|\bar{\rho} \circ y y_{\xi}(\bar{\rho} \circ y-\tilde{l} \circ y) y_{n, \xi}\right\|_{L^{1}} \\
& +\left\|\bar{\rho} \circ y y_{\xi}\left(\tilde{l} \circ y-\tilde{l} \circ y_{n}\right) y_{n, \xi}\right\|_{L^{1}}
\end{aligned}
$$




$$
\begin{aligned}
& +\left\|\bar{\rho} \circ y y_{\xi}\left(\tilde{l} \circ y_{n}-\bar{\rho} \circ y_{n}\right) y_{n, \xi}\right\|_{L^{1}} \\
\leq & \|\bar{\rho}\|_{L^{2}}\left(2\|\bar{\rho}-\tilde{l}\|_{L^{2}}+\left\|\tilde{l} \circ y_{n}-\tilde{l} \circ y\right\|_{L^{2}}\right) .
\end{aligned}
$$

Since $y_{n} \rightarrow y \in L^{\infty}(\mathbb{R})$ and $\tilde{l}$ is continuous with compact support, we obtain by Lebesque's dominated convergence theorem that $\tilde{l} \circ y_{n} \rightarrow \tilde{l} \circ y$ in $L^{2}(\mathbb{R})$. In particular, we can choose $n$ big enough so that $\left\|\bar{\rho} \circ y y_{\xi}\left(\bar{\rho} \circ y-\bar{\rho} \circ y_{n}\right) y_{n, \xi}\right\|_{L^{1}} \leq \varepsilon$. Since $\varepsilon$ can be chosen arbitrarily small we obtain in particular that

$$
\lim _{n \rightarrow \infty}\left\|\bar{\rho} \circ y y_{\xi}\left(\bar{\rho} \circ y-\bar{\rho} \circ y_{n}\right) y_{n, \xi}\right\|_{L^{1}}=0 .
$$

This immediately implies that also the second term tends to zero using $\bar{\rho}_{n} \circ y_{n}-$ $\bar{\rho}_{n} \circ y=\left(\bar{\rho}_{n} \circ y_{n}-\bar{\rho} \circ y_{n}\right)+\left(\bar{\rho} \circ y_{n}-\bar{\rho} \circ y\right)+\left(\bar{\rho} \circ y-\bar{\rho}_{n} \circ y\right)$. As far as the third (and the last) term is concerned, we can conclude as follows

$$
\begin{aligned}
\left\|\bar{\rho} \circ y_{n} y_{n, \xi}\left(\bar{\rho}_{n} \circ y-\bar{\rho} \circ y\right) y_{\xi}\right\|_{L^{1}} & \leq\left\|\bar{\rho} \circ y_{n} y_{n, \xi}\right\|_{L^{2}}\left\|\left(\bar{\rho}_{n} \circ y-\bar{\rho} \circ y\right) y_{\xi}\right\|_{L^{2}} \\
& \leq\|\bar{\rho}\|_{L^{2}}\left\|\bar{\rho}_{n}-\bar{\rho}\right\|_{L^{2}},
\end{aligned}
$$

which again tends to zero since by assumption $\bar{\rho}_{n} \rightarrow \bar{\rho} \in L^{2}(\mathbb{R})$. Hence all terms in (6.14) tend to 0 as $n \rightarrow \infty$ and therefore $\bar{r}_{n} \rightarrow \bar{r} \in L^{2}(\mathbb{R})$.

Finally we want to point out that when proving $U_{n, \xi} \rightarrow U_{\xi}$ in $L^{2}(\mathbb{R})$, one has that

$$
U_{n, \xi}^{2}=h_{n}-h_{n}^{2}-\bar{r}_{n}^{2},
$$

and a corresponding identity holds for $U_{\xi}$ which allows us to conclude as in [18, Proposition 5.1].

Lemma 6.5. Let $\left(u_{n}, \rho_{n}, \mu_{n}\right)$ be a sequence in $\mathcal{D}$ that converges to $(u, \rho, \mu)$ in $\mathcal{D}$. Then

$$
u_{n} \rightarrow u \text { in } L^{\infty}(\mathbb{R}), \quad \bar{\rho}_{n} \stackrel{*}{\rightarrow} \bar{\rho}, \quad k_{n} \rightarrow k \in \mathbb{R}, \quad \text { and } \quad \mu_{n} \stackrel{*}{\rightarrow} \mu .
$$

Proof. We denote by $X_{n}=\left(y_{n}, U_{n}, h_{n}, r_{n}\right)$ and $X=(y, U, h, r)$ the representative of $L\left(u_{n}, \rho_{n}, \mu_{n}\right)$ and $L(u, \rho, \mu)$ given by (4.6). For any $x \in \mathbb{R}$, there exist $\xi_{n}$ and $\xi$, not necessarily unique, such that $x=y_{n}\left(\xi_{n}\right)$ and $x=y(\xi)$. We set $x_{n}=y_{n}(\xi)$. We have

$$
u_{n}(x)-u(x)=u_{n}(x)-u_{n}\left(x_{n}\right)+U_{n}(\xi)-U(\xi)
$$

and

$$
\begin{aligned}
& \left|u_{n}(x)-u_{n}\left(x_{n}\right)\right|=\left|\int_{\xi}^{\xi_{n}} U_{n, \xi}(\eta) d \eta\right| \\
& \leq \sqrt{\xi_{n}-\xi}\left(\int_{\xi}^{\xi_{n}} U_{n, \xi}^{2} d \eta\right)^{1 / 2} \quad \text { (Cauchy-Schwarz) } \\
& \leq \sqrt{\xi_{n}-\xi}\left(\int_{\xi}^{\xi_{n}} y_{n, \xi} h_{n} d \eta\right)^{1 / 2} \quad \text { (from (3.23c)) } \\
& \leq \sqrt{\xi_{n}-\xi} \sqrt{\left|y_{n}\left(\xi_{n}\right)-y_{n}(\xi)\right|} \quad\left(\text { since } h_{n} \leq 1\right) \\
& =\sqrt{\xi_{n}-\xi} \sqrt{y(\xi)-y_{n}(\xi)} \\
& \leq \sqrt{\xi_{n}-\xi}\left\|y-y_{n}\right\|_{L^{\infty}}^{1 / 2} \text {. }
\end{aligned}
$$


From $|y(\xi)-\xi| \leq \mu(\mathbb{R})$, we get

$$
\left|\xi_{n}-\xi\right| \leq 2 \mu_{n}(\mathbb{R})+\left|y_{n}\left(\xi_{n}\right)-y_{n}(\xi)\right|=2\left\|h_{n}\right\|_{L^{1}}+\left|y(\xi)-y_{n}(\xi)\right|
$$

and, therefore, since $h_{n} \rightarrow h$ in $L^{1}(\mathbb{R})$ (because $h=U_{\xi}^{2}+\bar{r}^{2}-h \zeta_{\xi}$ ) and $y_{n} \rightarrow y$ in $L^{\infty}(\mathbb{R}),\left|\xi_{n}-\xi\right|$ is bounded by a constant $C$ independent of $n$. Then, (6.19) implies

$$
\left|u_{n}(x)-u_{n}\left(x_{n}\right)\right| \leq C\left\|y-y_{n}\right\|_{L^{\infty}}^{1 / 2} .
$$

Since $y_{n} \rightarrow y$ and $U_{n} \rightarrow U$ in $L^{\infty}(\mathbb{R})$, it follows from (6.18) and (6.20) that $u_{n} \rightarrow u$ in $L^{\infty}(\mathbb{R})$. By weak-star convergence, we mean that

$$
\lim _{n \rightarrow \infty} \int_{\mathbb{R}} \bar{\rho}_{n} \phi d x=\int_{\mathbb{R}} \bar{\rho} \phi d x
$$

for all continuous functions with compact support. It follows from (4.8b) that

$$
\int_{\mathbb{R}} \bar{\rho}_{n} \phi d x=\int_{\mathbb{R}} \bar{r}_{n} \phi \circ y_{n} d \xi \text { and } \int_{\mathbb{R}} \bar{\rho} \phi d x=\int_{\mathbb{R}} \bar{r} \phi \circ y d \xi .
$$

Since $y_{n} \rightarrow y$ in $L^{\infty}(\mathbb{R})$, the support of $\phi \circ y_{n}$ is contained in some compact which can be chosen independently of $n$ and, from Lebesgue's dominated convergence theorem, we have that $\phi \circ y_{n} \rightarrow \phi \circ y$ in $L^{2}(\mathbb{R})$. Hence, since $\bar{r}_{n} \rightarrow \bar{r}$ in $L^{2}(\mathbb{R})$,

$$
\lim _{n \rightarrow \infty} \int_{\mathbb{R}} \phi \circ y_{n} \bar{r}_{n} d \xi=\int_{\mathbb{R}} \phi \circ y \bar{r} d \xi
$$

and (6.21) follows from (6.22) Similarly one can show that $\mu_{n}$ converges weakly to $\mu$. Finally, that $k_{n}$ converges to $k$ is obvious by the definition of convergence in Eulerian coordinates.

\section{REFERENCES}

[1] A. Bressan and A. Constantin. Global conservative solutions of the Camassa-Holm equation. Arch. Ration. Mech. Anal., 183(2):215-239, 2007.

[2] A. Bressan and A. Constantin. Global dissipative solutions of the Camassa-Holm equation. Analysis and Applications, 5:1-27, 2007.

[3] R. M. Chen and Y. Liu. Wave breaking and global existence for a generalized two-component Camassa-Holm system. Inter. Math Research Notices, Article ID rnq118, 36 pages, 2010.

[4] M. Chen,, S.-Q. Liu, and Y. Zhang. A two-component generalization of the Camassa-Holm equation and its solutions. Lett. Math. Phys., 75:1-15, 2006.

[5] A. Constantin and R. I. Ivanov. On an integrable two-component Camassa-Holm shallow water system. Phys. Lett. A, 372(48):7129-7132, 2008.

[6] J. Escher, O. Lechtenfeld, and Z. Yin. Well-posedness and blow-up phenomena for the 2component Camassa-Holm equation. Discrete Contin. Dyn. Syst., 19(3):493-513, 2007.

[7] Y. Fu and C. Qu. Well posedness and blow-up solution for a new coupled Camassa-Holm equations with peakons. J. Math. Phys., 50:012906, 2009.

[8] K. Grunert, H. Holden, and X. Raynaud. Lipschitz metric for the Camassa-Holm equation on the line. Submitted 2010, arXiv:1010.0561

[9] K. Grunert, H. Holden, and X. Raynaud. Global conservative solutions of the Camassa-Holm equation for initial data with nonvanishing asymptotics. Submitted 2011, arXiv:1106.4125

[10] K. Grunert, H. Holden, and X. Raynaud. Lipschitz metric for the periodic Camassa-Holm equation. J. Differential Equations, 250(3):1460-1492, 2011.

[11] C. Guan, K. H. Karlsen, and Z. Yin. Well-posedness and blow-up phenomena for a modified two-component Camassa-Holm equation. In: Nonlinear Partial Differential Equations and Hyperbolic Wave Phenomena (eds. H. Holden and K. H. Karlsen), Amer. Math. Soc., Providence, pp. 199-220, 2010.

[12] C. Guan and Z. Yin. Global weak solutions for a modified two-component Camassa-Holm equation. Ann. I. H. Poincaré - AN 28:623-641, 2011. 
[13] C. Guan and Z. Yin. Global existence and blow-up phenomena for an integrable twocomponent Camassa-Holm shallow water system. J. Differential Equations, 248:2003-2014, 2010.

[14] G. Gui and Y. Liu. On the Cauchy problem for the two-component Camassa-Holm system. Math Z, 268:45-66, 2011.

[15] G. Gui and Y. Liu. On the global existence and wave breaking criteria for the two-component Camassa-Holm system. J. Func. Anal., 258:4251-4278, 2010.

[16] Z. Guo and Y. Zhou. On solutions to a two-component generalized Camassa-Holm equation. Studies Appl. Math., 124:307-322, 2010.

[17] D. Henry. Infinite propagation speed for a two component Camassa-Holm equation. Discrete Contin. Dyn. Syst. Ser. B, 12(3):597-606, 2009.

[18] H. Holden and X. Raynaud. Global conservative solutions of the Camassa-Holm equation - a Lagrangian point of view. Comm. Partial Differential Equations, 32(10-12):1511-1549, 2007.

[19] H. Holden and X. Raynaud. Dissipative solutions for the Camassa-Holm equation. Discrete Contin. Dyn. Syst. 24:1047-1112, 2009.

[20] P. A. Kuz'min. Two-component generalizations of the Camassa-Holm equation. Math. Notes, 81:130-134, 2007.

[21] K. Mohajer. A note on traveling wave solutions to the Camassa-Holm equation. J. Nonlin. Math. Phys., 16:117-125, 2009.

[22] O. G. Mustafa. On smooth traveling waves of an integrable two-component Camassa-Holm shallow water system. Wave Motion, 46:397-402, 2009.

[23] P. J. Olver and P. Rosenau. Tri-hamiltonian duality between solitons and solitary-wave solutions having compact support. Phys. Rev. B, 53(2):1900-1906, 1996.

[24] W. Tan and Z. Yin. Global dissipative solutions of a modified two-component Camassa-Holm shallow water system. J. Math. Phys., 52:033507, 2011.

[25] Y. Wang, J. Huang, and L. Chen. Global conservative solutions of the two-component Camassa-Holm shallow water system. Int. J. Nonlin. Science, 9:379-384, 2009.

Department of Mathematical Sciences, Norwegian University of Science and TechNOLOGY, 7491 TRONDHEIM, NORWAY

E-mail address: katring@math.ntnu.no

$U R L:$ http://www .math.ntnu.no/ katring/

Department of Mathematical Sciences, Norwegian University of Science and Technology, 7491 Trondheim, Norway, and Centre of Mathematics for Applications, UniverSity Oslo, 0316 OsLo, NoRWay

E-mail address: holden@math.ntnu.no

$U R L:$ http://www .math.ntnu.no/ holden/

Centre of Mathematics for Applications, University Oslo, 0316 Oslo, Norway

E-mail address: xavierra@cma.uio.no

URL: http://www.folk.uio.no/xavierra/ 